\title{
Rod Photoreceptor Activation Alone Defines the Release of Dopamine in the Retina
}

DOI:

10.1016/j.cub.2019.01.042

\section{Document Version}

Accepted author manuscript

Link to publication record in Manchester Research Explorer

\section{Citation for published version (APA):}

Pérez-Fernández, V., Milosavljevic, N., Allen, A., Vessey, K., Jobling, A., Fletcher, E., Breen, P., Morley, J., \& Cameron, M. (2019). Rod Photoreceptor Activation Alone Defines the Release of Dopamine in the Retina. Curr Biol, 29(5), 763-774.e5. https://doi.org/10.1016/j.cub.2019.01.042

\section{Published in:}

Curr Biol

\section{Citing this paper}

Please note that where the full-text provided on Manchester Research Explorer is the Author Accepted Manuscript or Proof version this may differ from the final Published version. If citing, it is advised that you check and use the publisher's definitive version.

\section{General rights}

Copyright and moral rights for the publications made accessible in the Research Explorer are retained by the authors and/or other copyright owners and it is a condition of accessing publications that users recognise and abide by the legal requirements associated with these rights.

\section{Takedown policy}

If you believe that this document breaches copyright please refer to the University of Manchester's Takedown Procedures [http://man.ac.uk/04Y6Bo] or contact uml.scholarlycommunications@manchester.ac.uk providing relevant details, so we can investigate your claim.

\section{OPEN ACCESS}




\section{CellPress}

\section{Sneak Peek}
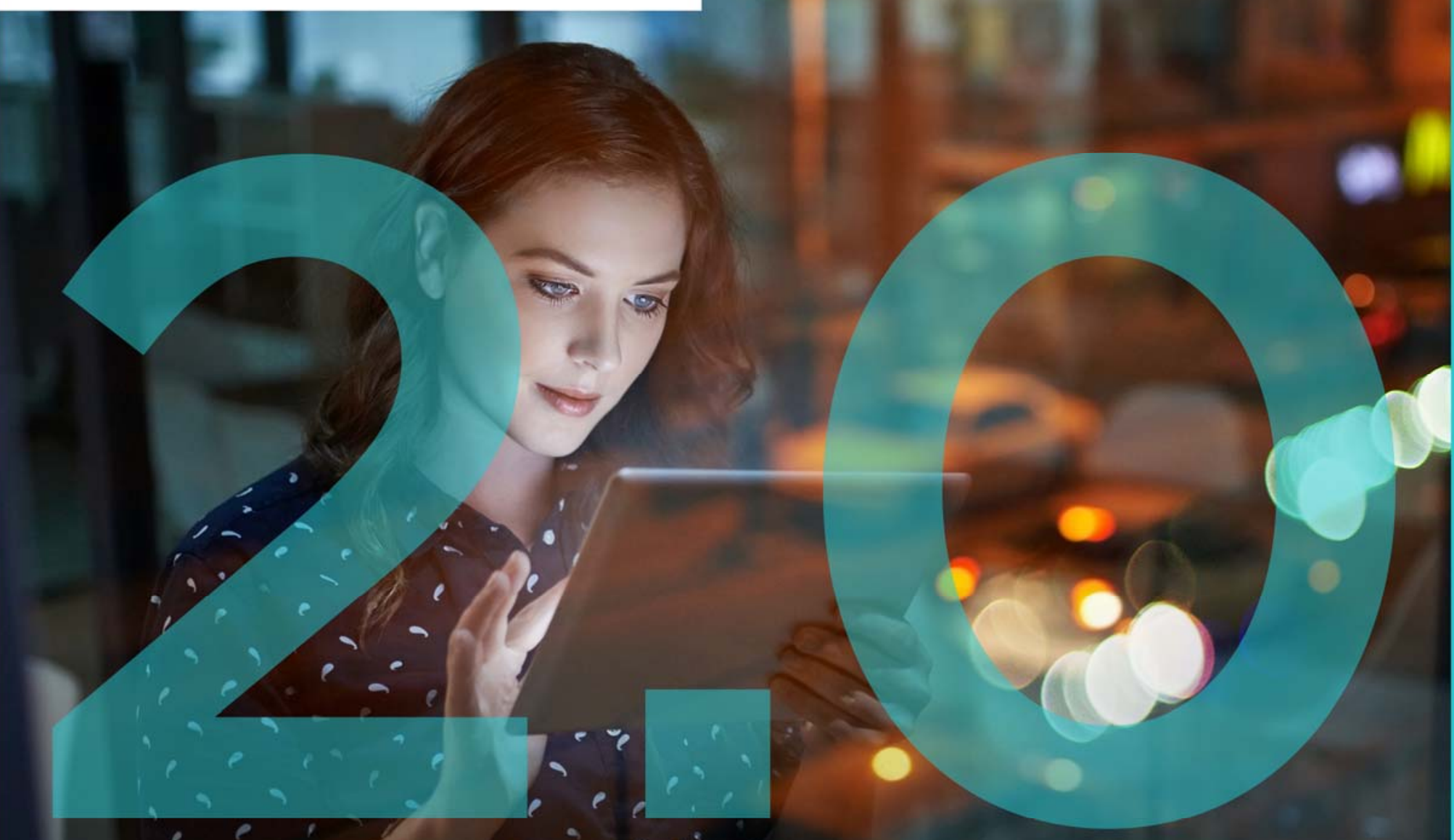

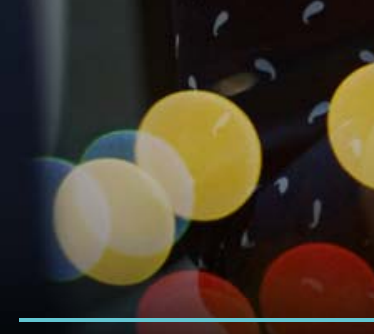

JOURNAL

\section{ARTICLE TITLE}

AUTHOR LIST
Current Biology

Rod photoreceptor activation alone defines the release of dopamine in the retina

Víctor Pérez-Fernández, Nina Milosavljevic, Annette E Allen, Kirstan A Vessey, Andrew I Jobling, Erica L Fletcher, John W Morley, Morven A Cameron 


\title{
Rod photoreceptor activation alone defines the release of dopamine in the retina
}

Víctor Pérez-Fernández ${ }^{1}$, Nina Milosavljevic ${ }^{2}$, Annette E Allen ${ }^{2}$, Kirstan A Vessey ${ }^{3}$, Andrew I Jobling ${ }^{3}$, Erica L Fletcher ${ }^{3}$, John W Morley ${ }^{1}$, Morven A Cameron ${ }^{1 *}$.

${ }^{1}$ School of Medicine, Western Sydney University, Sydney, Australia.

2 Department of Neuroscience and Experimental Psychology, Faculty of Biology, Medicine and Health, University of Manchester, UK.

${ }^{3}$ Department of Anatomy and Neuroscience, University of Melbourne, Melbourne, Australia.

${ }^{4}$ MARCS Institute, Western Sydney University, Sydney, Australia.

*Correspondence: m.cameron@westernsydney.edu.au

Keywords: retina; dopamine; rods; cones; melanopsin; adaptation; chemogenetics

\begin{abstract}
Retinal dopamine is released by a specialised subset of amacrine cells in response to light and has a potent influence on how the retina responds to, and encodes, visual information.

Here we address the critical question of which retinal photoreceptor is responsible for coordinating the release of this neuromodulator. While all three photoreceptor classes: rods, cones and melanopsin-containing retinal ganglion cells (mRGCs) have been shown to provide electrophysiological inputs to dopaminergic amacrine cells (DACs), we show here that the release of dopamine is defined only by rod photoreceptors. Remarkably, this rod signal coordinates both a suppressive signal at low intensities, and drives dopamine release at very bright light intensities. These data further reveal that dopamine release does not necessarily correlate with electrophysiological activity of dopaminergic amacrine cells and add to a growing body of evidence that rods define aspects of retinal function at very bright light levels.
\end{abstract}




\section{Introduction}

Dopamine is one of the most potent retinal neuromodulators, and its widespread functions define how retinal circuits encode visual information. Generally a signal for light-adaptation of the retinal circuitry, the effects of dopamine have been observed in growth and development, cell death, and myopia (reviewed [1, 2]). Therefore, there has been great interest in understanding the photoreceptor(s) and circuits responsible for coordinating its light-dependent release. Over recent years, the electrophysiological inputs to dopaminergic amacrine cells (DACs) have been defined in great detail, showing that all three photoreceptor classes: rods, cones and melanopsin-containing retinal ganglion cells (mRGCs), provide substantial input to these cells [3-7]. Conversely however, measurement of dopamine release in response to light does not correlate with electrophysiological input; while mRGC input alone can increase spiking of DACs this is not sufficient to drive dopamine release [8-10]. This finding matches recent discoveries in striatum-projecting midbrain dopaminergic neurons that display similar characteristics where dopamine release is not simply a passive read-out of midbrain cell firing [11]. It is proposed that dopamine can be locally released from axonal terminals in the striatum but the mechanism underlying this occurrence is unknown. The retina represents an ideal model to study the discontinuity between spiking and dopamine release given the well-defined and accessible nature of this tissue.

Retinal DACs reside in the middle of the retina where they give rise to an extensive dendritic/axonal plexus $[12,13]$. Dopamine release is thought to occur by volume transmission that diffuses throughout the entire depth of the retina to activate dopamine receptors on all known classes of neuron $[14,15]$. Here, we systematically investigate lightdependent release of dopamine in both wild-type and a variety of transgenic mice with altered photoreception and by using chemogenetics tools. Our data demonstrates that lightinduced dopamine release is unexpectedly high threshold, not being released until bright photopic light intensities ( $>400$ lux), and, surprisingly, that this threshold is defined by the level of rod function. Previously rods had been thought to only govern dim light vision, but recent reports have suggested a novel function for them under very bright-light $[16,17]$. Additionally, we reveal an inhibitory rod input that actively supresses dopamine release under dim- to mesopic light intensities. We conclude that a balance of excitatory and inhibitory inputs, driven by rod photoreceptors, defines the release of dopamine in the retina over 9 log units of light stimulation. Furthermore, we examine the mechanisms governing dopamine release and propose that it can be segregated within individual DACs to specific axonal/dendritic varicosities.

\section{Results}

\section{Light-induced dopamine release is high-threshold}

Measurement of dopamine release in neuronal tissue has been approached in a variety of ways over the years. Here we use two complementary techniques to assess dopamine release in the mouse retina (Fig $1 \mathrm{~A}$ ): $i)$ the ratio of one of the primary metabolites of dopamine (DA), 3,4-dihydroxyphenylacetic acid (DOPAC) to total DA (DOPAC:DA ratio) as an in vivo measure of dopamine turnover and ii) the release of dopamine from a dissected eye-cup (cornea, lens and iris removed) into an artificial media (artificial cerebrospinal fluid; aCSF) as an ex vivo measure. We were surprised to discover that a significant increase in light-induced dopamine 
turnover (DOPAC:DA ratio) or DA release (ex vivo), only occurred under bright photopic illumination (> $5 \log \mathrm{R}^{*} \operatorname{rod}^{-1} \mathrm{~s}^{-1} ; \sim 400 \mathrm{lux}$; Fig 1B\&C). When these two measures are directly compared after normalisation $\left(R / R_{\max }\right)$ and corrected for differences in retinal illuminance (see methods) this threshold was the same irrespective of the mode of measurement and lies $>6$ log units above the threshold for an electroretinogram (ERG) threshold response (Fig 1D). While such a high threshold for dopamine release was unexpected, the agreement between these two very different methods to assess dopamine release gives us confidence in the results. Moreover, the correlation of DOPAC:DA ratio to actual dopamine release from the retina further supports the utility of this indirect measure as a correlate of dopamine release.

\section{Dim light actively suppresses dopamine release}

The lowest irradiance point in Fig 1B was dim red light $\left(\sim 0.017 \log \mathrm{R}^{*} \operatorname{rod}^{-1} \mathrm{~s}^{-1}\right)$ and was generally used as the "dark" condition in accordance with previous work $[10,18,19]$, though rods will be activated by this stimulus. This point corresponds to $<1 \mathrm{~min}$ of light exposure during removal of the retinal tissue, as opposed to a $1 \mathrm{hr}$ white pulse at all other irradiances. However, when retinae were extracted under infrared light (IR; $-2.5 \log R^{*} \operatorname{rod}^{-1} \mathrm{~s}^{-1}$ ) over a similar time-scale, an increase in dopamine turnover was observed (Fig 1E). These data indicate that the dim red light, presumably acting via rods at these intensities, is actively suppressing dopamine release.

\section{Circadian rhythmicity of light-induced dopamine release is not observed}

As light-induced dopamine turnover has been previously shown to be released in a circadian manner, with greater turnover at subjective midnight (CT18) as opposed to subjective midday (CT6) [10] we compared irradiance responses under these conditions (Fig 1F). Contrary to previous findings, no circadian differences were observed at any of the irradiances tested. This is likely due to the fact that animals were anaesthetised while light-pulsed, rather than free-moving in their home-cage. While we have previously shown that anaesthesia does not change the amplitude of the response, we find that it greatly reduces intra-individual variability [20]. We hypothesize that anaesthesia allows a more standardized light pulse to be applied to the animals rather than when they are free-moving in their home cage. All further experiments were performed within $2 \mathrm{hrs}$ of CT6. No circadian difference in dark adapted dopamine release was observed in agreement with previous studies on C57BL/6 mice that lack melatonin [21].

\section{Cone input is not required for light-induced dopamine release}

While previous data suggests that rods predominately drive dopamine release [10], this is hard to rectify with the fact that the threshold of activation is at least 6 log units above that of rods making cones or mRGCs a more likely origin for this input. We first assessed cone input to light-induced dopamine release in a mouse model lacking cone phototransduction $\left(\right.$ Gnat $\left.{ }^{A 518 G}\right)$. These animals have morphologically normal cones and little cellular reorganisation at the ages used ( $2-3$ months) but lack any measurable cone response [22]. In comparison to wild-type animals, Gnat ${ }^{A 518 G}$ mice showed indistinguishable light-induced dopamine release that could be fit with the same curve (Fig 2A; extra-sum-of-squares $F$ test, $\mathrm{P}=0.70$; also observed using the ex vivo protocol Fig S1B), suggesting cone function is not necessary to drive this response or set the threshold. Furthermore, dim red light produced the same level of suppression when compared to infrared meaning cones are not driving this 
suppression in dim light (Fig 2B). In agreement with wild-types, Gnat2 ${ }^{A 518 G}$ animals did not show any circadian differences in irradiance response (Fig 2C).

To determine the function of rods under the intensities that elicit dopamine release we recorded light-adapted flash electroretinograms from Gnat2 ${ }^{A 518 G}$ and wild-type animals under two different adapting backgrounds. Michaelson contrast was constant for both conditions (0.99) and intensities of the background and flash ( $2 \mathrm{~ms})$ were controlled by the addition of neutral density filters. Background A (Fig 2D) is below the dopamine release threshold (3.3 log $\mathrm{R}^{*}$ rod $\left.^{-1} \mathrm{~s}^{-1}\right)$ and $G n a t 2^{A 518 G}$ animals display a measurable rod response, although it is smaller and slower than that of the wild-type (blue; Fig 2E). However, background $B$ is just above the dopamine release threshold (5.3 $\log R^{*} \operatorname{rod}^{-1} \mathrm{~s}^{-1}$ ) and Gnat2 ${ }^{A 518 G}$ do not display any noticeable response, whereas the wild-type response is very similar to that recorded under background A (Fig 2F). While rods are not "silent" under these conditions, these data suggest that they do not contribute significantly to the transient response to light flashes in the presence of a background above the dopamine release threshold.

Given that some mouse models of cone inactivation have been shown to be incomplete, due the second-messenger promiscuity of opsins [23], we assessed the impact of cone photoreception on light induced dopamine release in an alternative way utilising mice expressing the human L-cone opsin in place of the mouse M-cone opsin $\left(O p n 1^{m w R}\right)[24]$. Activation of cones in these animals can be spectrally segregated from that of rods and mRGCs. We designed a red $(624 \mathrm{~nm})$ light stimulus that activates L-cones above the "threshold" for dopamine release, (see effective photon calculations in Fig S2) but is below threshold for rods and mRGCs, allowing us to ascertain if it is cones that are determining this threshold. We found that this red stimulus was not sufficient to drive light-induced dopamine release in the Opn1 $1^{m w R}$ mouse (although a positive trend was observed; Fig 2G), indicating that cones are not setting the threshold for full amplitude light-induced dopamine release. We then applied a red stimulus that was matched for rod sensitivity with that of a blue pulse (434 nm) in wild-type animals and found that red light can drive significant dopamine release but only when it is bright enough to stimulate rods above the dopamine threshold (Fig $2 \mathrm{H}$ ). Taken together these experiments indicate that a photoreceptor with a spectral sensitivity matching that of rod opsin determines the dopamine release threshold.

As cone input can be transient due to adaptation [25], and because previous data has shown that input to DACs can be enhanced by using a flickering light $[26,27]$, we compared dopamine turnover in response to a flickering or continuous white light stimulus just below the dopamine release threshold $\left(4.6 \log \mathrm{R}^{*} \operatorname{rod}^{-1} \mathrm{~s}^{-1}\right)$ in wild-type animals. Flickering light did not enhance the response to white light at this intensity (Fig 2I) suggesting that the light input to DACs to elicit dopamine release is specific to the intensity of light and cannot be enhanced by summing many transient ON inputs.

\section{mRGCs are neither necessary nor sufficient to drive light-induced dopamine release}

We assessed $\mathrm{mRGC}$ input to light-induced dopamine release in a variety of ways. Firstly, using rodless coneless $(r d / r d)$ mice we completed an irradiance response curve and found that dopamine turnover is not significantly altered at any light intensity (Fig 3A). Furthermore, mice lacking melanopsin $\left(O p n 4^{c r e / c r e}\right)$ show irradiance response curves that are 
indistinguishable from wild-type animals (Fig 3B; extra-sum-of squares F-test, $p=0.88$; also observed using the ex vivo protocol Fig S1). Additionally, while Opn4 $4^{\text {cre/cre }}$ mice showed normal suppression of dopamine turnover by dim red light compared to infrared, no difference between the two was observed in the $r d / r d$ animals (Fig $3 C$ ), further implicating rods in driving this suppression.

The retinae of the $r d / r d$ animals undergo significant neural reorganisation following photoreceptor degeneration and although mRGC electrophysiological input to DACs is maintained in these animals [3] we investigated if dopamine could actually be released from DACs in $r d / r d$ mice. Inclusion of high extracellular concentration potassium (15 mM as opposed to $3 \mathrm{mM}$ ) in the aCSF to elicit neuronal depolarisation caused significant dopamine release both under dark and light conditions (Fig 3D). These results show that while the $r d / r d$ retina is capable of releasing dopamine, it cannot be triggered by mRGC input.

Finally, to assess mRGC input to DACs in a non-degenerate retina we turned to chemogenetic activation of mRGCs in the Opn $4^{\text {cre/+ }}$ retina. The clozapine- $N$-oxide (CNO) sensitive hM3D Gqcoupled receptor fused to mCherry was introduced via intravitreal injection of pAAV2-hSynDIO-hM3D(Gq)-mCherry. Expression was confirmed via immunohistochemistry as described previously [28]. mRGC activation by CNO was confirmed in vivo by pupil constriction (under IR $>950 \mathrm{~nm}$ ) and c-fos activation of mCherry positive cells (Fig 3E), and in vitro by measuring $m R G C$ spiking via patch-clamp recording of mCherry expressing cells (Fig S3). No significant increase in dopamine turnover occurred when hM3Dq expressing Opn4 $4^{\text {cre/+ }}$ animals were injected intraperitoneally (under dim red light) with $5 \mathrm{mg} / \mathrm{kg} \mathrm{CNO}$ and maintained for $1 \mathrm{hr}$ in the dark (Fig 3F). As c-fos activation of TH-expressing cells has been shown to correlate with dopamine release, it was assessed in tandem. While $68.8 \% \pm 3.7$ (mean \pm SEM) of $\mathrm{TH}^{+}$ amacrines in wild-type light-pulsed (5.3 $\log \mathrm{R}^{*} \operatorname{rod}^{-1} \mathrm{~s}^{-1} ; 90 \mathrm{~min}$ ) retinae expressed c-fos, only $4.7 \% \pm 1.7$ of $\mathrm{TH}^{+}$cells expressed $\mathrm{c}$-fos in $\mathrm{CNO}$ activated $\mathrm{hM} 3 \mathrm{Dq}$ expressing retinae (Fig 3G; no c-fos positive cells were found in dim red/control conditions). As we know that mRGC activity is upregulated in a consistent and prolonged way: in vivo (pupil remains constricted up to $2 \mathrm{hrs}$; Fig S3A) and in vitro (prolonged increase in spiking; Fig S3B-D), this indicates that even with persistent activation of $\mathrm{mRGCs}$ by $\mathrm{hM} 3 \mathrm{Dq}$ receptors for $1 \mathrm{hr}$, dopamine is not released from DACs.

Taken together with previous results, these data indicate that it is rod photoreceptor input that defines dopamine release in the retina at all light intensities, while cones and mRGCs are neither necessary nor sufficient to drive this response.

\section{Pharmacological block of light-induced dopamine release}

We next aimed to identify the major rod pathways that convey light information to DACs. To do this we utilised the ex vivo method that allows pre-treatment of retinae with pharmacological agents prior to light pulsing. We found that the glycine receptor blocker strychnine $(10 \mu \mathrm{M})$ reduced basal dopamine release under dim red light but did not inhibit light-induced dopamine release (Fig $4 A$ ). The $G_{A B A} / c$ receptor blocker picrotoxin $(50 \mu \mathrm{M})$ caused significant dopamine release, to light levels, in dim red but did not augment the light response (Fig $4 \mathrm{~A})$. These data suggest that the bright light input may be relieving a constitutive GABAergic inhibition of DACs, as previously suggested by others [29, 30]. In agreement with previous reports, the mGluR6 receptor agonist L-AP4 (50 $\mu \mathrm{M}$; blocking ON bipolar input) 
reduced dopamine release both in dim red and bright light conditions suggesting the origin of light-induced dopamine release occurs in the outer retina (Fig 4B). In contrast, while the AMPA/kainite receptor blocker CNQX $(10 \mu \mathrm{M})$ blocked light-induced dopamine release, it had no effect on basal levels (Fig 4B). Surprisingly, we found that the NMDA receptor blocker AP5 $(10 \mu \mathrm{M})$ significantly reduced, although did not entirely eliminate, light-induced dopamine release (Fig $4 \mathrm{~B}$ ). These data indicate that the light-input must travel through ON bipolar cells and be mediated by AMPA/kainite and, to a large part, NMDA receptor input at some point in the circuit.

\section{Gap-junctions mediate input to dopaminergic amacrines}

It is known that rod input to ON ganglion cells relies heavily on gap-junction connectivity between All amacrine cells and cone ON bipolars cells (primary rod pathway) and between rods and cone photoreceptors (secondary rod pathway)[31, 32]. Since light-induced dopamine release is blocked by L-AP4, our current understanding of the circuitry suggests the rod signal travels though one, or both, of these pathways. Using the pan-connexin blocker meclofenamic acid (MFA; $200 \mu \mathrm{M}$ ) we see that the majority of light induced dopamine release is attenuated in both wild-type and Gnat2 ${ }^{A 518 G}$ animals (Fig 4C). However, significant release is maintained in both genotypes suggesting the existence of a gap-junction independent pathway driven by rods. When comparing the magnitude of dopamine release between wildtypes and Gnat $2^{A 518 G}$ animals we see that light-induced absolute amplitude is reduced when cone function is eliminated. This is contrary to the in vivo turnover of dopamine (Fig $2 \mathrm{~A}$ ) and may reflect morphological changes in these animals that limits the diffusion of dopamine out of the retina. However, threshold for release when amplitudes were normalised was not significantly different between wild-types and Gnat $2^{A 518 G}$ animals using the ex vivo protocol (Fig S1).

\section{Spiking and synaptic inputs combine to cause light-induced dopamine release}

To further investigate the mechanisms governing dopamine release we assessed the impact of synaptic input and/or spiking. DACs, unlike some amacrine cells, fire action potentials and dopamine release is correlated with spiking [33]. Addition of the voltage-gated sodium channel (Nav) blocker tetrodotoxin (10 $\mu \mathrm{M}$ TTX) abolished light-induced dopamine release and significantly reduced basal levels under dim red light confirming that spiking is necessary for dopamine release (Fig 5A). Conversely, increasing neuronal depolarisation, and spiking, in the retina as a whole with $15 \mathrm{mM} \mathrm{KCl}$ did not increase dopamine release under dim red light (although a positive trend was observed), but significantly augmented light-induced release (Fig 5B). These data suggest that both spiking and light-induced synaptic input are important to promote dopamine release. Furthermore, addition of $\mathrm{Mg}^{2+}$-free solution to $r d / r d$ retinae to allow activation of NMDA receptors without coincident depolarisation caused a significant increase in dopamine release in both $\mathrm{dim}$ and bright-light conditions in $\mathrm{rd} / \mathrm{rd}$ retinae (Fig $5 \mathrm{C}$ ). These data implicate a glutamate input to NMDA receptors that must be paired with spiking to produce significant dopamine release.

\section{Duration response characteristics of dopamine release}

To further characterise dopamine release dynamics using both in vivo and ex vivo protocols, we completed a duration-response curve for the brightest light intensity $\left(6.8 \log \mathrm{R}^{*} \operatorname{rod}^{-1} \mathrm{~s}^{-1}\right.$; Fig 6A). Animals were light pulsed in vivo with different duration pulses up to $1 \mathrm{hr}$ and then maintained in the dark for 1-60 min until their retinae were extracted. The DOPAC:DA ratio 
showed a rapid increase within $30 \mathrm{sec}$ and a peak at $1 \mathrm{~min}$ that reduced and recovered within the following $10 \mathrm{~min}$ in an oscillatory manner. By 15 min dopamine turnover plateaued and remained at this level for the remainder of the hour. After cessation of the light, the DOPAC:DA ratio showed a transient increase (although not significant) and then declined quickly and was significantly reduced within $10 \mathrm{~min}$, and back to baseline levels after $1 \mathrm{hr}$. These data suggest that the DOPAC:DA ratio gives an "instantaneous" measure of dopamine release within the retina with an integration time of less than 2 min.

A duration-response curve was also completed for the ex vivo conditions over $15 \mathrm{~min}$ and these results are overlayed with in vivo DOPAC:DA ratio in Fig 6B. Ex vivo dopamine release showed a slower initial increase, likely reflecting the rate of DA diffusion out of the retina into the surrounding aCSF. Taken together, these data indicate that while in vivo and ex vivo measures of dopamine release can report similar intensity release profiles, they display quite different temporal release properties.

\section{Mathematical model of dopamine turnover}

Taking this duration-response curve into account, we aimed to construct a mathematical model to describe dopamine release in response to a step of bright light. Since the light input to DACs is affected by gap-junction contribution (Fig 4C) we must consider the fact that dopamine itself alters gap junction connectivity between many cell types in the retina [3437]. Consequently, if dopamine itself is reducing the light input to DACs by closing gap junctions we might expect that dopamine release would exhibit an oscillatory release profile, i.e. more release when dopamine concentration is low and gap junctions are open, and less release when dopamine is high and gap junctions are closed. In fact, we do see a glimpse of this negative feedback in our time-course of the DOPAC:DA ratio (Fig 6A), with dopamine peaking at $1 \mathrm{~min}$, reducing to a trough at $5 \mathrm{~min}$ and then returning to this peak at $15 \mathrm{~min}$. However, the response to light off is somewhat different with a counterintuitive initial peak in dopamine release at $\sim 60$ s and gradual exponential decline over time. While these occurrences could be caused by mechanisms intrinsic to DOPAC production itself (monoamine oxidase enzyme activity or dopamine uptake efficiency) the simplest mathematical model we found to elicit the observed response is illustrated in Fig 6C\&D. A similar dopamine turnover profile (Fig 6C (ii)) can be replicated by applying a step light input (Fig 6C (i)) low-pass filtered ( $\tau=20 \mathrm{~s}$ ) minus a negative feedback component (Fig 6C (iii)) and a direct inhibitory component (Fig 6C (iii)). The negative feedback is the effect of dopamine release itself on the amplitude of the light input low-passed ( $\tau=100 \mathrm{~s}$ ) and delayed $60 \mathrm{~s}$, which declines slowly over time $(\tau=1000 \mathrm{~s}$; Fig 6D(iii)). The direct inhibitory component comes directly from the light input (Fig 6D(iv)), however, while this direct inhibition is included in both the light and dark responses its impact is only clearly observable as the transient increase in dopamine release following transition to dark (3600 s; $60 \mathrm{~min}$ ). The model also shows that dopamine release exhibits hysteresis, whereby the transfer function in response to light is different to the dark response $(\tau=1000 \mathrm{~s})$, suggesting that a reduction in retinal dopamine in the dark is slower than the increase in the light.

The model therefore fits well with the rest of our data that suggest rods both inhibit and stimulate dopamine release via separate circuits and suggests that dopamine itself provides an inhibitory feedback on its own release. It appears that the direct inhibition we observe under dim-light (Fig 1E) can be overcome at bright light intensities and we propose that a 
balance of excitation and inhibition driven by rods alone defines the amplitude of dopamine release in the mouse retina.

\section{Discussion}

This study has identified the profile of dopamine release in the mouse retina over a large range of light intensities. This release appears to be exclusively defined by rod input and shows a U-shape, with more dopamine released under dark and bright conditions and less dopamine released under dim and mesopic conditions.

The high threshold of dopamine release ( $>5 \log ^{*} \operatorname{rod}^{-1} \mathrm{~s}^{-1}$; equating to $\sim 400$ lux) was unexpected given that it is driven by a rod input as we might expect rod photoreceptors to be already saturated at these light intensities. However, our data definitively show that dopamine release is not dependent on cone photoreceptors or mRGCs. In the last few years rods have been shown to function at intensities far above what was originally assumed [16, 17] so it is plausible that bleaching adaptation within rod photoreceptors preserves their function at such bright light intensities. The light intensities that elicit dopamine release, while bright, are normal physiological irradiances that would be experienced outdoors during the day-time (ranging from $400-200,000$ lux). The threshold for release corresponds to the switch from mesopic (rod and cone shared) function of the retina, to purely photopic (cone only)[38]. Indeed, rod responses cannot be recorded via ERG in the Gnat2 ${ }^{A 518 G}$ retina when a background above the dopamine threshold is applied (5.3 $\mathrm{R}^{*} \operatorname{rod}^{-1} \mathrm{~s}^{-1}$; Fig $\left.2 \mathrm{~F}\right)$, although a significant ERG can be recorded with a background below this threshold $\left(3.3 \mathrm{R}^{*}\right.$ rod $^{-1} \mathrm{~s}^{-1}$; Fig $2 \mathrm{E}$ ) suggesting that this is around the light intensity where rods are no longer useful to the system. Based on these data, it is likely that widespread dopamine release is causing removal of this "inoperable" rod input from the cone pathway to optimize the retina for vision under day-time light intensities.

Furthermore, our data imply that a dim light input to rods is actively supressing dopamine release. However, this suppression must occur over a very short time scale as animals were only exposed to the red head torch for $<1 \mathrm{~min}$ before the retina was removed and snap frozen in the dark. Block with L-AP4 further decreases dopamine release in dim red light suggesting that suppression at these intensities does not occur via ON bipolar mediated pathways. It is possible that basal dopamine release under dim light intensities is mediated by a separate circuit to that of light-induced dopamine release, possibly acting via a direct rod to OFF bipolar cell synapse [39]. Dopamine has been shown to actually sensitize rod ON bipolar function at dark and very dim light intensities [40] implying that as light intensity moves from around rod threshold to $2-3$ log units above, dopamine release is reduced causing graded desensitization of rod ON bipolar cells.

One of the most noteworthy conclusions from our data is that dopamine release does not match electrophysiological input to TH labelled amacrine cells [3-6]. Specifically, and controversially, we comprehensively show that the input from mRGCs does not elicit dopamine release (Fig 3). This is further supported by our result that L-AP4 abolishes lightinduced dopamine release in the wild-type retina (Fig 4B), as the electrophysiological input from mRGCs to TH amacrines persists in the presence of L-AP4 [3]. It is important to 
distinguish the fact that neuronal activation (specifically spiking) does not necessarily equal neurotransmitter release, two distinct processes that are often used interchangeably in the literature. This is perhaps more specific to dopamine release as it has been suggested in striatum-projecting midbrain dopaminergic neurons that dopamine can be released via a local presynaptic mechanism that triggers dopamine release directly from axonal varicosities in the striatum, bypassing activity in dopaminergic somas [41]. We propose that this situation is mirrored in the retina where dopamine release can be triggered in axonal/dendritic varicosities by microcircuits comprising both presynaptic inputs and vesicle release in the same structure (Fig 7). Certainly, dopamine is thought to be mainly released from DAC processes [42], whereas all electrophysiological inputs have been described at the soma. Electrotonic isolation of distal neurites is likely given the lack of voltage-gated sodium channels (Nav) found in DAC processes [43], and there is certainly precedent for this mechanism in the retinal A17 amacrine cells that are able to operate more than 100 independent microcircuits within the same cell [44]. In this scenario, while the basal firing rate of the neuron at the soma undoubtedly has an influence on the physiology of the cell, dopamine release could be triggered locally from each individual varicosity. This type of mechanism would be useful in a DAC to limit dopamine release to the vicinity of the light (synaptic) input. If this were not the case, activation of a dopaminergic cell soma would elicit dopamine release throughout the entire network of processes (up to $1.6 \mathrm{~mm}$ in diameter [13]). This hypothesis is supported by our data that simply increasing spiking of DACs by applying high $\mathrm{KCl}$ does not cause significant dopamine release, whereas high $\mathrm{KCl}+$ light gives an augmented response. Interestingly, $r d / r d$ animals show the opposite effect where an increase in spiking (or NMDA channel disinhibition) does cause significant release. However, these animals show significant synaptic reorganisation [45] and spontaneous activity [46] that alter their functional circuitry. Finally, if changes in spiking of DACs are not directly correlated with dopamine release, what are the function of these inputs? Since TH-labelled cells have been shown to release both GABA and ATP $[47,48]$, electrophysiological inputs from mRGCs and cones may be more important to these neurotransmitter systems.

NMDA receptor activation appears necessary for the majority of light-induced dopamine release. Extra-synaptic NMDA receptors have been shown to augment All and A17 amacrine cell light responses, specific to rod pathways [49]. Therefore, it may be at the rod ON bipolar to All or A17 amacrine synapse where NMDA activation greatly increases the rod input at bright light intensities through glutamate spill over. The finding that both spiking and synaptic input is required to promote dopamine release suggests that glutamate activation of NMDA channels, only in combination with spiking, causes dopamine release. GluR1/GluR4 and NR1 are certainly found on the spines of $\mathrm{TH}$-labelled cell varicosities [12] providing a possible mechanism to spatially segregate dopamine release to discrete parts of a single DAC.

The most parsimonious mathematical model we determined to explain dopamine release in response to rod input indicates that release is determined by a balance between excitatory and inhibitory pathways. In this model, "lights on" provides both excitation and direct inhibition of dopamine release. Interestingly, this direct inhibition, incorporated in the model to explain the transient increase in dopamine release at light offset, is of a similar magnitude to the reduction in dopamine release caused by dim red light (Fig 1E). The model suggests that this direct inhibition is still present during light-induced dopamine release, but that it is overwhelmed by the excitatory input. We must be careful here to point out that "excitation" 
and "inhibition" in the model relates to increased or decreased dopamine release rather than the specific neurotransmitters involved in these processes e.g. an "excitatory input" could occur via a reduction in GABAergic input. Furthermore, the model predicts that the "lights on" excitatory input is reduced by the release of dopamine itself as a negative feedback. This likely relates to the effect of dopamine on retinal circuitry, possibly via a reduction in gap junction conductance. This negative feedback from dopamine attenuates significantly over time. While we do not know the mechanism governing this reduction, it may reflect desensitisation of dopamine receptors. However, since we are examining the dopamine turnover in the entire retina and we do not have spatial information, it may be that the initial large amplitude release of dopamine from the DACs reaches a high concentration in the INL/IPL that becomes diffused within the whole retina over time. Therefore, determining the spatial and temporal dynamics of dopamine diffusion is important to describe the mechanism of this negative feedback. Newly developed genetically encoded dopamine sensors would be ideal to analyse this process in more detail in future studies $[50,51]$.

\section{Summary of dopamine release in the mouse retina}

Taking this data together, we present a summary figure for the possible circuitry governing dopamine release in response to light stimulation (Fig 7). Under dark conditions, tonic spiking occurs that causes release of a basal level of dopamine; there is no inhibition or excitation at the level of dopaminergic varicosities as the light signal is absent. Under dim scotopic and mesopic light stimuli there is an increase in GABA inhibition to varicosities, likely driven through the OFF system. Glutamate release is also increased and stimulates synaptic AMPA/kainite receptors, but does not outweigh the inhibition causing an overall reduction in basal dopamine release. Under bright light conditions both inhibition and excitation are increased to the varicosity but due to glutamate spill over to extra-synaptic NMDA receptors the level of excitation tips the balance and allows a large release of dopamine. While it is likely that the precise circuitry mediating dopamine release is much more complex than that depicted above, all our data points toward the regulation of dopamine release in the retina relying on the balance between inhibition and excitation driven exclusively by rod photoreceptors.

The retina represents an ideal model tissue in which to study dopamine release mechanisms in the CNS given that the inputs causing release can be precisely defined and localised. Future work examining both the spatial and temporal aspects of light-induced dopamine release in the retina will likely be of great importance in elucidating the mechanisms governing release from dopaminergic cells in the rest of the brain. 


\section{STAR $\star$ Methods}

Key Resources Table

\begin{tabular}{|c|c|c|}
\hline REAGENT or RESOURCE & SOURCE & IDENTIFIER \\
\hline \multicolumn{3}{|l|}{ Antibodies } \\
\hline $\begin{array}{l}\text { Donkey Anti-Mouse lgG }(\mathrm{H}+\mathrm{L}) \\
\text { Fluorescein (FITC) }\end{array}$ & $\begin{array}{l}\text { Jackson ImmunoResearch } \\
\text { Laboratories, INC. }\end{array}$ & $\begin{array}{l}\text { Cat\#715-095-151, } \\
\text { RRID:AB_2335588 }\end{array}$ \\
\hline $\begin{array}{l}\text { Donkey Anti-Rabbit IgG }(\mathrm{H}+\mathrm{L}) \\
\text { Rhodamine (TRITC) }\end{array}$ & $\begin{array}{l}\text { Jackson ImmunoResearch } \\
\text { Laboratories, INC. }\end{array}$ & $\begin{array}{l}\text { Cat\#711-025-152, } \\
\text { RRID:AB_2340588 }\end{array}$ \\
\hline Goat Anti-Rabbit $(\mathrm{H}+\mathrm{L})$ Alexa Fluor 488 & Life Technologies & $\begin{array}{l}\text { Cat\#A-11008, } \\
\text { RRID:AB_143165 }\end{array}$ \\
\hline Rabbit Monoclonal Anti-c-Fos (9F6) & Cell Signalling Technology & $\begin{array}{l}\text { Cat\#2250, } \\
\text { RRID:AB_2247211 }\end{array}$ \\
\hline $\begin{array}{l}\text { Sheep Polyclonal Anti-Tyrosine } \\
\text { Hydroxylase }\end{array}$ & Merck Millipore & $\begin{array}{l}\text { Cat\#657012, RRID: } \\
\text { AB_696697 }\end{array}$ \\
\hline \multicolumn{3}{|l|}{ Bacterial and Virus Strains } \\
\hline pAAV-hSyn-DIO-hM3D(Gq)-mCherry & Addgene & Cat\#44361-AAV2 \\
\hline \multicolumn{3}{|c|}{ Chemicals, Peptides, and Recombinant Proteins } \\
\hline $\begin{array}{l}\text { (2R)-amino-5-phosphonovaleric acid } \\
\text { (AP5) }\end{array}$ & Cayman Chemical & $\begin{array}{l}\text { Cat\#14539, } \\
\text { CAS:79055-68-8 }\end{array}$ \\
\hline $\begin{array}{l}\text { 6-cyano-7-nitroquinoxaline-2,3-dione } \\
\text { (CNQX) }\end{array}$ & Cayman Chemical & $\begin{array}{l}\text { Cat\#14618, } \\
\text { CAS:115066-14-3 }\end{array}$ \\
\hline Alexa Fluor 488 Hydrazide & Invitrogen & Cat\#A10436 \\
\hline Biocytin & Life Technologies & $\begin{array}{l}\text { Cat\# } \quad \text { B1592, } \\
\text { CAS:576-19-2 }\end{array}$ \\
\hline Clozapine $\mathrm{N}$-oxide (CNO) & Hellobio & $\begin{array}{l}\text { Cat\#HB6149, } \\
\text { CAS:5786-21-0 }\end{array}$ \\
\hline DOPAC-d5 & Cambridge Isotope Laboratories & $\begin{array}{l}\text { Cat\#DLM-2499-PK, } \\
\text { CAS:60696-39-1 }\end{array}$ \\
\hline Dopamine-d4 & CDN Isotopes & $\begin{array}{l}\text { Cat\#D-1540, } \\
\text { CAS:203633-19-6 }\end{array}$ \\
\hline Hypromellose & Alcon & GenTeal \\
\hline Ketamine & Troy, Illium & CAS:6740-88-1 \\
\hline $\begin{array}{l}\text { L-2-amino-4-phosphonobutyric acid } \\
\text { (L-AP4) }\end{array}$ & Abcam & $\begin{array}{l}\text { Cat\#ab120002, } \\
\text { CAS:23052-81-5 }\end{array}$ \\
\hline Meclofenamic Acid (MFA) & Sigma-Aldrich & $\begin{array}{l}\text { Cat\#M4531-5G, } \\
\text { CAS:6385-02-0 }\end{array}$ \\
\hline Phenylephrine & Bausch and Lomb & $\mathrm{N} / \mathrm{A}$ \\
\hline Picrotoxin & Sigma-Aldrich & $\begin{array}{l}\text { Cat\#P1675, } \\
\text { CAS:124-87-8 }\end{array}$ \\
\hline Salts, acids and bases & Sigma-Aldrich & $\mathrm{N} / \mathrm{A}$ \\
\hline Streptavidin & Life Technologies & $\begin{array}{l}\text { Cat\#S11223, } \\
\text { CAS:9013-20-1 }\end{array}$ \\
\hline Strychnine & Sigma-Aldrich & $\begin{array}{l}\text { S0532, CAS:57-24- } \\
9\end{array}$ \\
\hline Tetrodotoxin (TTX) & TOCRIS & $\begin{array}{l}\text { Cat\#1078, } \\
\text { CAS:4368-28-9 }\end{array}$ \\
\hline
\end{tabular}




\begin{tabular}{|c|c|c|}
\hline Tropicamide & Alcon & Mydriacyl 1\% \\
\hline Xylazine & Troy, Illium & CAS:7361-61-7 \\
\hline \multicolumn{3}{|l|}{ Experimental Models: Organisms/Strains } \\
\hline Mouse: C57BL/6J & ARC, Canning Vale, Australia & JAX: 000664 \\
\hline Mouse: Gnat2 $2^{A 518 G}$ & $\begin{array}{l}\text { (Jobling, Vessey, Waugh, Mills, \& } \\
\text { Fletcher, 2013) }\end{array}$ & N/A \\
\hline Mouse: Opn $4^{\mathrm{Cre} /+} / \mathrm{Opn} 4^{\mathrm{Cre} / \mathrm{Cre}}$ & (Ecker et al., 2010) & $\mathrm{N} / \mathrm{A}$ \\
\hline Mouse: Opn $1^{m w R}$ & (Smallwood et al., 2003) & $\mathrm{N} / \mathrm{A}$ \\
\hline Mouse: $r d / r d\left(\mathrm{C} 57 \mathrm{BL} / 6 \mathrm{~J}-\mathrm{Pde} 6 \mathrm{~b}^{\mathrm{rd1}-2 \mathrm{~J}} / \mathrm{J}\right)$ & The Jackson Laboratory & Stock no: 004766 \\
\hline \multicolumn{3}{|l|}{ Software and Algorithms } \\
\hline GraphPad Prism 7 & GraphPad Software & $\mathrm{N} / \mathrm{A}$ \\
\hline LabChart & ADinstruments & $\mathrm{N} / \mathrm{A}$ \\
\hline MassLynx 4.1 & Waters MassLynx Software & $\mathrm{N} / \mathrm{A}$ \\
\hline MATLAB & MathWorks & $\mathrm{N} / \mathrm{A}$ \\
\hline pClamp 10 & Molecular Devices & $\mathrm{N} / \mathrm{A}$ \\
\hline Metavue v7.8.4.0 & Molecular Devices & $\mathrm{N} / \mathrm{A}$ \\
\hline OceanView 1.6.7 & Ocean Optics & $\mathrm{N} / \mathrm{A}$ \\
\hline \multicolumn{3}{|l|}{ Other } \\
\hline 0.9 ND filters & LEE filters & Cat\#211 0.9ND \\
\hline Collimated LED & Thorlabs & Cat\#MCWHLP1 \\
\hline Blue filter (434/17nm) & Semrock & $\begin{array}{l}\text { Cat\#FF01-434/17- } \\
25\end{array}$ \\
\hline Red filter (624/40nm) & Edmund Optics & Cat\#67035 \\
\hline Head torch 8 LED & VARTA & $\mathrm{N} / \mathrm{A}$ \\
\hline Head torch red filter & LEE filters & $\begin{array}{l}\text { Cat\#787 Marius } \\
\text { Red }\end{array}$ \\
\hline IR Goggles & Night Vision Australia & Cat\#D2MVSL \\
\hline Photometer & Irradian & Cat\#L202 Macam \\
\hline SAD Lamp & Litebook & $\begin{array}{l}\text { Cat\#Edge } \\
\text { Lightbox }\end{array}$ \\
\hline Spectroradiometer & Ocean Optics & $\begin{array}{l}\text { Cat\#USB2000+XR1 } \\
\text {-ES }\end{array}$ \\
\hline
\end{tabular}

Contact for Reagent and Resource Sharing

Further information and requests for resources should be directed to and will be fulfilled by the Lead Contact, Morven A. Cameron (m.cameron@westernsydney.edu.au).

\section{Experimental Model and Subject Details}

Mice

Animal care was in accordance with either the Australian Code for the Care and Use of Animals for Scientific Purposes or the UK Animals, Scientific Procedures, Act 1986 (c-fos DREADDs experiment). Protocols were approved and monitored by the Western Sydney University Animal Care and Ethic Committee, project numbers: A11900 and A12402. Wildtype C57BL/6J (ARC, Canning Vale, Australia), Gnat2 ${ }^{A 518 G}$ (C56BL/6J backcrossed), rd/rd (C57BL/6J backcrossed), Opn $1^{\text {mwR }}, O p n 4^{\text {cre/cre }}$ and Opn $4^{\text {cre/t }}$ (mixed C57BL/6 and 129sv) mice 
were bred on site. Offspring (both male and female) 60 - 100 days were used. Animals were maintained under a $12 \mathrm{hr}$ light: dark cycle at 300 lux illumination during the daytime.

\section{Method Details}

\section{Light pulsing}

For in vivo experiments, mice were handled under either dim red or infrared light, anaesthetized with an intraperitoneal injection of $70 \mathrm{mg} / \mathrm{kg}$ Ketamine $+7 \mathrm{mg} / \mathrm{kg}$ Xylazine (Troy Laboratories), and their eyes dilated with Tropicamide 1\% (Alcon) before light pulsing. Control animals were not anaesthetised and were maintained in complete darkness, we have previously shown that anaesthesia does not affect dopamine release under dark conditions [20]. After light pulsing, or control dark conditions, mice were sacrificed by cervical dislocation and eyes enucleated (under the same lighting conditions as the pulse, or red/infrared light) and retinae extracted through a lateral incision along the ora serrata by adding pressure to both sides of the cut. Each retina was put into a $0.5 \mathrm{~mL}$ plastic tube and immediately frozen in liquid nitrogen. The tubes containing the retinae were stored at $-80{ }^{\circ} \mathrm{C}$ until analysed. As the response to light stimulation was the same at $15 \mathrm{~min}$ as $1 \mathrm{hr}$ (Figure 6A) pulsing was shortened to $15 \mathrm{~min}$ as described in the corresponding figure legends.

For ex vivo experiments, after overnight adaptation to the dark, mice were sacrificed by cervical dislocation under dim red light, eyes enucleated, and the cornea and lens removed. The remaining eyecup was placed, with the retina facing up, into a $0.5 \mathrm{~mL}$ plastic tube with $40 \mu \mathrm{L}$ of oxygen-bubbled, HEPES-buffered aCSF ( $\mathrm{NaCl} 140$, HEPES 10, $\mathrm{KCl} 3, \mathrm{NaH}_{2} \mathrm{PO}_{4} 1.25$, $\mathrm{CaCl}_{2} 2$ and $\mathrm{MgCl}_{2}$ 1, Glucose $10 \mathrm{mM}$ ) at room temperature, and either light-pulsed or kept in the dark. All pharmacological agents were dissolved in HEPES-buffered aCSF to a final concentration as stated. Tissue was incubated in each agent for $>10 \mathrm{~min}$ (30 min for meclofenamic acid) prior to light-pulsing or dark incubation. The supernatant aCSF was then removed and frozen in liquid nitrogen and stored at $-80{ }^{\circ} \mathrm{C}$ until analysed. For ex vivo pharmacology experiments dark and light controls were completed in parallel as intraexperiment variability was much lower than inter-experiment variability.

Retinae from the in vivo experiment were homogenized using a pellet pestle cordless motor inside a $40 \mu \mathrm{L}$ solution consisting of: $1 \%$ formic acid (Sigma-Aldrich), $0.5 \mathrm{mM}$ ascorbic acid (Sigma-Aldrich) and $10 \mathrm{ng} / \mathrm{mL}$ the deuterated standards of dopamine- $d 4$ (CDN Isotopes) and DOPAC- $d 5$ (Cambridge Isotope Laboratories). Supernatant from the ex vivo experiments was made up to a solution of $1 \%$ formic acid, $0.5 \mathrm{mM}$ ascorbic acid, $10 \mathrm{ng} / \mathrm{mL}$ deuterated standards. Then, for both in vivo and ex vivo preparations, samples were centrifuged at 14,000 r.p.m. for $10 \mathrm{~min}$ at $4{ }^{\circ} \mathrm{C}$ and the supernatant placed in clear glass total recovery vials (Waters) for chromatographic analysis.

\section{UHPLC-MS/MS}

Ultra-high performance liquid chromatography and tandem mass spectrometry was performed as described in [20]. Briefly, samples were analysed on a Waters Xevo TQ-MS triple quadrupole mass spectrometer, fitted with an electrospray ionization source. The desolvation gas flow $\left(\mathrm{N}_{2}\right)$ was $800 \mathrm{~L} / \mathrm{h}$, desolvation temperature was $450^{\circ} \mathrm{C}$, cone gas $0 \mathrm{~L} / \mathrm{h}$, and collision gas ( $\mathrm{Ar}$ ) flow of $0.15 \mathrm{~mL} / \mathrm{min}$, which gave a collision cell pressure of $2.6 \times 10^{-3} \mathrm{mbar}$. In positive ion mode the capillary voltage was set at $1.2 \mathrm{kV}$ and in negative mode $1.0 \mathrm{kV}$. Liquid chromatography was performed using a Waters Acquity UPLC, working at a flow rate of 0.20 $\mathrm{mL} / \mathrm{min}$. A Waters Acquity UPLC BEH C18 column of $1.7 \mu \mathrm{m}$ particle size and dimensions 2.1 
$\times 150 \mathrm{~mm}$ was used, operating at $40^{\circ} \mathrm{C}$. Solvent A consisted of $0.1 \%$ formic acid in ultrapure water and solvent $B$ was $0.1 \%$ formic acid in methanol. A 20 min run was employed, commencing at $5 \%$ B for $1 \mathrm{~min}$, increasing linearly to $100 \%$ B by $10 \mathrm{~min}$, then returning immediately to $5 \%$ B at $15 \mathrm{~min}$. The sample manager was kept at $4{ }^{\circ} \mathrm{C}$ and injections of $10 \mu \mathrm{L}$ were made in full loop mode. Retention times for dopamine and dopamine- $d_{4}$ were 2.1 and $2.04 \mathrm{~min}$ respectively and were analysed in positive ion mode. DOPAC and DOPAC- $d_{5}$ were 5.09 and 5.01 min respectively and were analysed in negative ion mode. Dopamine only (ex vivo) or dopamine and DOPAC (in vivo) for each retina were quantified and dopamine per retina (ex vivo) or DOPAC: dopamine ratio (in vivo) is expressed as mean \pm S.E.M.

\section{Light calculations}

Light stimuli for in vivo and ex vivo irradiance response curves were provided by an LED array (EDGE SAD Lightbox; Litebook) delivered from above the animal/eye preparation. Irradiance

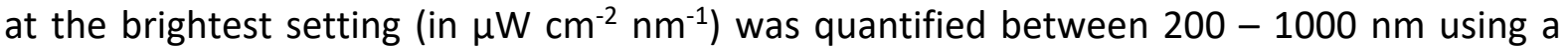
spectroradiometer fitted with a cosine diffuser (USB2000+XR1-ES; Ocean Optics) measured at eye level. Irradiance at each wavelength was then converted to photons $\mathrm{cm}^{-2} \mathrm{~s}^{-1} \mathrm{~nm}^{-1}$. Total effective photons $\mathrm{cm}^{-2} \mathrm{~s}^{-1}$ for each photoreceptor was calculated taking into account the spectral sensitivity of each photoreceptor type using Govardovskii nomograms [52] for a vitamin A1 based opsin of $\lambda_{\max }$ as follows: rod $-498 \mathrm{~nm}, \mathrm{M}$-cone $-508 \mathrm{~nm}$, S-cone $-360 \mathrm{~nm}$, human L-cone $-556 \mathrm{~nm}$, Opn4 - $480 \mathrm{~nm}$. Photoisomerisations per rod, per second ( $\mathrm{R}^{*}$ rod $^{-1}$ $\mathrm{s}^{-1}$ ) were calculated by converting effective rod photons $\mu \mathrm{m}^{-2} \mathrm{~s}^{-1}$, assuming a rod collecting

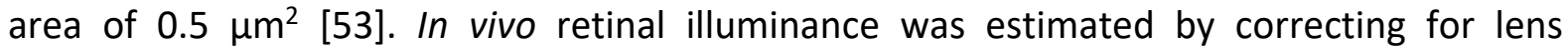
transmission [54], whereas the lens was removed in the ex vivo preparation, so transmission was assumed to be $100 \%$ at all wavelengths. The eye aperture was estimated to be $\sim 3.2 \mathrm{~mm}^{2}$ (fully dilated pupil) in vivo, and $\sim 8 \mathrm{~mm}^{2}$ ex vivo (cornea and iris removed) giving a multiplication factor of 0.18 and 0.45 respectively (assuming a retinal area of $17.8 \mathrm{~mm}^{2}$ [55]. Generally light is expressed as $\mathrm{R}^{*}$ rod $^{-1} \mathrm{~s}^{-1}$ (corrected for either in vivo or ex vivo) however, illuminance at the level of the cornea in lux was calculated by Ocean View software and independently confirmed via a photometer (L202 Macam; Irradian) and is used alongside R* $\operatorname{rod}^{-1} \mathrm{~s}^{-1}$ to provide context to readers. Neutral density film (0.9ND; LEE filters) was applied to the lamp to reduce intensity without changing spectral composition.

Electroretinogram (ERG) stimuli, the red and blue light-pulses (Fig 2E) and flicker stimuli were provided by a single high-power collimated LED (MCWHLP1; Thorlabs) and delivered either via ganzfeld illumination (ERG), or from above the animal/eye prep. Red and blue light-pulses employed a $624 \mathrm{~nm}$ and a $434 \mathrm{~nm}$ filter. Irradiance was precisely controlled by a combination of the voltage output for the LED and neutral density filters (Edmund Optics).

"Dim-red" for in vivo conditions involved exposing the animal to an LED headtorch filtered with red film (> $650 \mathrm{~nm}$; Marius Red; LEE Filters) for $<1 \mathrm{~min}$ to isolate the retina, whereas "dim-red" for the ex vivo prep involved dissection of the tissue under a dissection scope illuminator filtered with the same red film that took 3 mins. Infrared conditions were only employed in vivo using IR goggles (NVAD2MV; Night Vision Australia), the IR illuminator on these goggles was $>800 \mathrm{~nm}$. Spectra of all stimuli were measured as described above, and $R^{*}$ $\operatorname{rod}^{-1} \mathrm{~s}^{-1}$ calculated. Experiments involving Opn1 ${ }^{m w R}$ animals employed only IR goggles for "dark" conditions. 


\section{Chemogenetics}

Intravitreal injections of AAV2-hSyn-DIO-hM3Dq-mCherry vector $\left(2.3 \times 10^{\wedge} 13\right.$ genomic particles/ml; The UNC Vector Core) were performed as previously reported $[28,56]$ and employed hyaluronan lyase and heparinase III ( $0.2 \mathrm{U}$ each) to maximise retinal penetration (total volume $2.5 \mathrm{ul}$ injected over $1 \mathrm{~min}$ ). Control eyes underwent the same procedure, with injections including glycosydic enzymes but with the virus replaced by vehicle (PBS). Mice were allowed at least 6 weeks to recover before being used in in vivo studies.

Dopamine release: $\mathrm{Opn} 4^{\mathrm{Cre} /+}$ mice expressing hM3Dq in the right eye only, were dark-adapted overnight. CNO $(5 \mathrm{mg} / \mathrm{kg})$ was administered i.p. under dim-red light and kept for $1 \mathrm{hr}$ in the dark. Both retinae were removed under dim-red light and the left eye served as a control for the hM3Dq expressing eye. Pupil constriction in response to CNO was quantified 2 weeks prior using the same methods and visualised using an IR webcam (DCS-930L; D-Link) illuminated by a custom-made IR LED array (>950 nm). Animals with pupil constriction of less than $80 \%$ in response to CNO injection were excluded.

c-fos: Opn $4^{\mathrm{Cre} /+}$ mice expressing hM3Dq were dark-adapted overnight, CNO $(5 \mathrm{mg} / \mathrm{kg})$ was administered i.p. under dim red light and mice were kept for 90 min in the dark. After 90 minutes of treatment mice were deeply anaesthetized with i.p. injection of urethane $(2.2 \mathrm{~g} / \mathrm{kg}$; $30 \% \mathrm{w} / \mathrm{v}$; Sigma-Aldrich, UK) and perfused transcardially with $0.9 \%$ saline followed by $4 \%$ paraformaldehyde. Wild-type animals underwent the same conditions, but without CNO injection and one group was light-pulsed, free-moving in their home cage with LED light (5.3 $\left.\log R^{*} \operatorname{rod}^{-1} s^{-1}\right)$.

\section{Immunohistochemistry}

Immunohistochemistry on retinal sections was performed as previously described [28, 56]. cFos staining employed rabbit anti-c-Fos primary (Cell Signaling 9F6; 1:250), and Alexa 488 conjugated goat anti-rabbit secondary (Life Technologies; 1:300) antibodies. TH staining employed sheep anti-TH primary (Merck AB1542; 1:300), and Alexa 647 conjugated donkey anti-sheep (Life Technologies, 1:300) antibodies. Endogenous mCherry fluorescence revealed expression of hM3Dq-mCherry in hM3Dq-expressing mice. Images were collected on an Olympus BX51 upright microscope using a [20x / 0.5 UPlan FLN] objective and captured using a [Coolsnap ES2 camera (Photometrics)] through Metavue v7.8.4.0 software (Molecular Devices). More than 15 retinal sections were analysed per each retina.

\section{Electroretinogram}

Animals were anaesthetised under IR illumination with i.p. injection of ketamine $(70 \mathrm{mg} / \mathrm{kg})$ and xylazine $(7 \mathrm{mg} / \mathrm{kg})$ and maintained with s.c. injection of ketamine $(72 \mathrm{mg} / \mathrm{kg})$ and xylazine $(5 \mathrm{mg} / \mathrm{kg}$ ). Mydriatics (tropicamide 1\% \& phenylephrine $2.5 \%)$ and hypromellose solution (0.5\%) (Alcon, Belgium; Bausch and Lomb, UK) were applied to the recording eye prior to application of an active electrode consisting of a platinum wire (100 $\mu \mathrm{m}$ thickness) embedded in a contact lens made from Aclar transparent film (ProSciTech, Australia). A needle reference electrode was inserted approximately $5 \mathrm{~mm}$ from the base of contralateral eye, distal enough to preclude signal contamination. A platinum wire bite bar was used to support the head and to act as ground. Electrode setup and injection of anaesthetic agents was conducted under IR light, the experimental setup was then covered with a light-tight faraday cage. Electrodes were connected to a PowerLab 26T where they were differentially amplified x 2000, band- 
pass filtered 0.5 to $300 \mathrm{~Hz}$ (sampling rate $10 \mathrm{kHz}$ ) and digitized to a Mac running LabChart Software (ADinstuments). Core body temperature was maintained by placing animals in a custom-made hose coil connected to a constant temperature water source. In all cases electrode stability was confirmed for 10 min prior to recording.

All mice were long-term dark adapted $(>12 \mathrm{hr}$ ) prior to recording ERGs. Flash and background stimuli were white light from a collimated LED (described above) reflected in a custom made Ganzfeld dome and attenuated with neutral density filters (Edmund Optics). For dark-adapted irradiance response measures a series of $10 \mathrm{~ms}$ flashes were delivered at $1 \mathrm{~Hz}$ for the lowest intensity, up to $0.01 \mathrm{~Hz}$ at the brightest intensity with repetitions decreasing accordingly. An average trace was then generated for each irradiance. The $b$-wave amplitude was quantified by summing the absolute a-wave (unfiltered) amplitude and the b-wave amplitude when filtered (low pass $20 \mathrm{~Hz}$ ) to remove the influence of oscillatory potentials. Simple sigmoidal curves were fitted to the irradiance response data for both amplitude and implicit time.

$$
y=V_{\min }+\frac{V_{\max }-V_{\min }}{1+10^{\left(\log E C_{50}-x\right)}}
$$

In order to take account of the initial manifestation of the a-wave on the ERG waveform, the b-wave amplitude responses were fitted with separate simple sigmoidal functions at low and high irradiances [57]. Light-adapted ERGs were recorded (after $20 \mathrm{~min}$ adaptation to the background) in response to 20 repeats of a 2 ms flash of 0.99 Michaelson contrast (controlled via LED voltage). Light intensity of the background and flash were controlled by filtering with neutral density filters (Edumund Optics). ERGs were recorded through the middle of the subjective day (CT2-10). To express ERG responses in comparison to continuous light in the dopamine release experiment, irradiance was converted to $\mathrm{R}^{*} \operatorname{rod}^{-1} \mathrm{~s}^{-1}$ as described above. Therefore, in the dark-adapted irradiance response curve the maximum intensity flash ( $6 \mathrm{log}$ $\left.R^{*} \operatorname{rod}^{-1} \mathrm{~s}^{-1}\right)$ elicits 4 Log $R^{*} \operatorname{rod}^{-1}\left(\sim 1.6 \log \mathrm{cd}-\mathrm{s} / \mathrm{m}^{2}\right)$.

\section{Modelling}

To estimate the dynamics of dopamine release in response to light, we developed a simplified model in Simulink (Matlab, The Mathworks). While the number of empirical data points available to describe the light response curves are limited, they are sufficient to highlight some key features on the basis of which the model is created. Independent model elements are described as transfer functions in the Laplace domain. Indeed, as all transfer functions used were first order low pass filters, each function is a variant of

$$
G(s)=\frac{\text { Output }}{\text { Input }}=\frac{1}{\tau s+1}
$$

where $\tau$ is the time constant and $\mathrm{s}$ is a complex number, $s=a+j \omega$. The Laplace transform $G=G(s)$ of any time function $f=f(t)$ are related by the equation

$$
G(s)=\int_{0}^{\infty} f(t) e^{-s t} d t
$$

Gain factors, time delays, signal limits and simple arithmetic functions complete the model. 


\section{Electrophysiology}

Animals were euthanized using cervical dislocation, eyes enucleated, cut along the ora serrata, and placed in artificial cerebrospinal fluid (ACSF) containing (mM): $\mathrm{NaCl}$ 125, $\mathrm{NaHCO}_{3} 25, \mathrm{KCl} 3, \mathrm{NaH}_{2} \mathrm{PO}_{4} 1.25, \mathrm{CaCl}_{2} 2$ and $\mathrm{MgCl}_{2}$ 1, Glucose 10, at room temperature (RT) within $1 \mathrm{~min}$ of euthanasia under dim red light conditions. Whole-mount retinae were isolated and cut into 4 pieces. As needed, retinal pieces were then transferred to the recording chamber where they were infused with carbogen $\left(5 \% \mathrm{CO}_{2} / 95 \% \mathrm{O}_{2}\right)$ bubbled ACSF at a rate of 2-3 ml/min. A tissue anchor (harp) made of platinum with nylon threads ( $\sim 500 \mu \mathrm{m}$ apart) was used to keep the tissue in place. Whole-cell current clamp recordings were made from mCherry expressing retinal ganglion cells with patch electrodes of resistances 5.0-8.0 $\mathrm{M} \Omega$. Electrodes were filled with (mM): $120 \mathrm{KMeSO}_{4}, 10 \mathrm{KCl}, 0.008 \mathrm{CaCl}_{2}, 0.5 \mathrm{EGTA}, 1 \mathrm{MgCl}$, $10 \mathrm{HEPES}, 4 \mathrm{ATP}-\mathrm{Na}_{2}$, and $0.5 \mathrm{GTP}-\mathrm{Na}_{3}$, adjusted to $\mathrm{pH} 7.4$ with $\mathrm{KOH}$. Alexa Fluor $488 \mathrm{Hydraz}$ (70 $\mu \mathrm{M}$, Invitrogen A10436) and biocytin hydrazide (0.3\%, Life Technologies B1592) were also included in the pipette solution for morphological analysis. The series resistance $\left(R_{S}\right)$ was monitored throughout the experiments and was in the range of 10-30 $\mathrm{M} \Omega$. Data was acquired with a Multiclamp 700B amplifier, low-pass filtered at $10 \mathrm{kHz}$ at the amplifier output and digitized by a Digidata $1440 \mathrm{~A}$ at $50 \mathrm{kHz}$ and conveyed to a computer running pClamp 10 (Molecular Devices). CNO was dissolved in aCSF and applied either via whole-bath application or puffed from a $3 \mathrm{M} \Omega$ patch pipette approximately $10 \mu \mathrm{m}$ from the cell body.

Following recording, retinal pieces were fixed in 4\% PFA for $1 \mathrm{hr}$, then washed thoroughly with Tris $0.1 \mathrm{M} \mathrm{pH}$ 7.4. Alexa488 tagged streptavidin (Life Technologies, S11223) was applied in Tris-Triton ( $0.3 \%$, Sigma X-100) at a dilution of 1:200 for 24 hrs before retinae were mounted on glass slides and coverslipped with Vectashield (Vectorlabs H-1000).

\section{Quantification and Statistical Analysis}

Chromatograms for dopamine, DOPAC and their respective deuterated standards were analysed with MassLynx and are presented as DOPAC: dopamine ratio as means \pm SEM as noted in the text and figure legends. For in vivo experiments (DOPAC: dopamine) each $n$ represents the mean between the ratios of both retinae from the same animal, whereas for ex vivo experiments ( $\mathrm{pg}$ dopamine/retina) each $n$ represents a single retina since once extracted can undergo different treatments or serve as dark controls. One-way analysis of variance (ANOVA) with Dunnett's or Tukey's multiple comparison test was applied when comparing different light or pharmacological conditions to dark or untreated control, and Student's test was applied when comparing between two light conditions within the same treatment. All graphs and statistical analysis were performed with GraphPad Prism 7. Statistical significance was defined as a $\mathrm{p}$ value smaller than 0.05 .

\section{Acknowledgements}

We would like to acknowledge the support of the Mass Spectrometry Facility at Western Sydney University for providing use of the instruments, equipment, and LC solvents to complete these experiments. We particularly thank Dr David Harman and Meena Mikhael for their input to the optimisation of the experiments.

\section{Author Contributions}


M.A.C. conceived of the project, designed the experiments and wrote the manuscript. V.P.F. completed all of the in vivo and ex vivo experiments involving dopamine release and quantification by UHPLC-MS/MS. A.E.A. and N.M. designed and completed the chemogenetics experiments. P.B. designed and completed the mathematical modelling. K.A.V., A.I.J. and E.L.F. provided the Gnat2 ${ }^{A 518 G}$ mouse and provided feedback on all experiments. J.W.M. provided equipment and reagents and was involved with the design and interpretation of all experiments. All authors provided feedback on the manuscript.

\section{DECLARATION OF INTERESTS}

The authors declare no competing interests.

\section{References}

1. Witkovsky, P. (2004). Dopamine and retinal function. Doc Ophthalmol 108, 17-40.

2. Chakraborty, R., and Pardue, M.T. (2015). Molecular and Biochemical Aspects of the Retina on Refraction. Prog Mol Biol Transl Sci 134, 249-267.

3. Zhang, D.Q., Wong, K.Y., Sollars, P.J., Berson, D.M., Pickard, G.E., and McMahon, D.G. (2008). Intraretinal signaling by ganglion cell photoreceptors to dopaminergic amacrine neurons. Proc Natl Acad Sci U S A 105, 14181-14186.

4. Prigge, C.L., Yeh, P.T., Liou, N.F., Lee, C.C., You, S.F., Liu, L.L., McNeill, D.S., Chew, K.S., Hattar, S., Chen, S.K., et al. (2016). M1 ipRGCs Influence Visual Function through Retrograde Signaling in the Retina. J Neurosci 36, 7184-7197.

5. Qiao, S.N., Zhang, Z., Ribelayga, C.P., Zhong, Y.M., and Zhang, D.Q. (2016). Multiple cone pathways are involved in photic regulation of retinal dopamine. Sci Rep 6, 28916.

6. Zhao, X., Wong, K.Y., and Zhang, D.Q. (2017). Mapping physiological inputs from multiple photoreceptor systems to dopaminergic amacrine cells in the mouse retina. Sci Rep 7, 7920.

7. Newkirk, G.S., Hoon, M., Wong, R.O., and Detwiler, P.B. (2013). Inhibitory inputs tune the light response properties of dopaminergic amacrine cells in mouse retina. J Neurophysiol 110, 536-552.

8. Frucht, Y., Vidauri, J., and Melamed, E. (1982). Light activation of dopaminergic neurons in rat retina is mediated through photoreceptors. Brain Res 249, 153-156.

9. Nir, I., and luvone, P.M. (1994). Alterations in light-evoked dopamine metabolism in dystrophic retinas of mutant rds mice. Brain Res 649, 85-94.

10. Cameron, M.A., Pozdeyev, N., Vugler, A.A., Cooper, H., luvone, P.M., and Lucas, R.J. (2009). Light regulation of retinal dopamine that is independent of melanopsin phototransduction. Eur J Neurosci 29, 761-767.

11. Berke, J.D. (2018). What does dopamine mean? Nat Neurosci 21, 787-793.

12. Fasoli, A., Dang, J., Johnson, J.S., Gouw, A.H., Fogli Iseppe, A., and Ishida, A.T. (2017). Somatic and neuritic spines on tyrosine hydroxylase-immunopositive cells of rat retina. J Comp Neurol 525, 1707-1730.

13. Zhang, D.Q., Stone, J.F., Zhou, T., Ohta, H., and McMahon, D.G. (2004). Characterization of genetically labeled catecholamine neurons in the mouse retina. Neuroreport 15, 1761-1765.

14. Nguyen-Legros, J., Versaux-Botteri, C., and Vernier, P. (1999). Dopamine receptor localization in the mammalian retina. Molecular neurobiology 19, 181-204. 
15. Bjelke, B., Goldstein, M., Tinner, B., Andersson, C., Sesack, S.R., Steinbusch, H.W., Lew, J.Y., He, X., Watson, S., Tengroth, B., et al. (1996). Dopaminergic transmission in the rat retina: evidence for volume transmission. Journal of chemical neuroanatomy 12, 37-50.

16. Pahlberg, J., Frederiksen, R., Pollock, G.E., Miyagishima, K.J., Sampath, A.P., and Cornwall, M.C. (2017). Voltage-sensitive conductances increase the sensitivity of rod photoresponses following pigment bleaching. J Physiol 595, 3459-3469.

17. Tikidji-Hamburyan, A., Reinhard, K., Storchi, R., Dietter, J., Seitter, H., Davis, K.E., Idrees, S., Mutter, M., Walmsley, L., Bedford, R.A., et al. (2017). Rods progressively escape saturation to drive visual responses in daylight conditions. Nat Commun 8 , 1813.

18. Nir, I., Haque, R., and luvone, P.M. (2000). Diurnal metabolism of dopamine in the mouse retina. Brain Res 870, 118-125.

19. Megaw, P.L., Boelen, M.G., Morgan, I.G., and Boelen, M.K. (2006). Diurnal patterns of dopamine release in chicken retina. Neurochem Int 48, 17-23.

20. Pérez-Fernández, V., Harman, D.G., Morley, J.W., and Cameron, M.A. (2017). Optimized Method to Quantify Dopamine Turnover in the Mammalian Retina. Analytical chemistry 89, 12276-12283.

21. Doyle, S.E., Grace, M.S., Mclvor, W., and Menaker, M. (2002). Circadian rhythms of dopamine in mouse retina: the role of melatonin. Vis Neurosci 19, 593-601.

22. Jobling, A.I., Vessey, K.A., Waugh, M., Mills, S.A., and Fletcher, E.L. (2013). A naturally occurring mouse model of achromatopsia: characterization of the mutation in cone transducin and subsequent retinal phenotype. Invest Ophthalmol Vis Sci 54, 33503359.

23. Allen, A.E., Cameron, M.A., Brown, T.M., Vugler, A.A., and Lucas, R.J. (2010). Visual responses in mice lacking critical components of all known retinal phototransduction cascades. PLoS ONE 5, e15063.

24. Smallwood, P.M., Olveczky, B.P., Williams, G.L., Jacobs, G.H., Reese, B.E., Meister, M., and Nathans, J. (2003). Genetically engineered mice with an additional class of cone photoreceptors: implications for the evolution of color vision. Proc Natl Acad Sci U S A 100, 11711.

25. Lall, G.S., Revell, V.L., Momiji, H., Al Enezi, J., Altimus, C.M., Guler, A.D., Aguilar, C., Cameron, M.A., Allender, S., Hankins, M.W., et al. (2010). Distinct contributions of rod, cone, and melanopsin photoreceptors to encoding irradiance. Neuron 66, 417428.

26. Mills, S.L., Xia, X.B., Hoshi, H., Firth, S.I., Rice, M.E., Frishman, L.J., and Marshak, D.W. (2007). Dopaminergic modulation of tracer coupling in a ganglion-amacrine cell network. Vis Neurosci 24, 593-608.

27. Weiler, R., Baldridge, W.H., Mangel, S.C., and Dowling, J.E. (1997). Modulation of endogenous dopamine release in the fish retina by light and prolonged darkness. Vis Neurosci 14, 351-356.

28. Milosavljevic, N., Allen, A.E., Cehajic-Kapetanovic, J., and Lucas, R.J. (2016). Chemogenetic Activation of ipRGCs Drives Changes in Dark-Adapted (Scotopic) Electroretinogram. Invest Ophthalmol Vis Sci 57, 6305-6312.

29. Feigenspan, A., Gustincich, S., Bean, B.P., and Raviola, E. (1998). Spontaneous activity of solitary dopaminergic cells of the retina. J Neurosci 18, 6776-6789. 
30. Marshburn, P.B., and luvone, P.M. (1981). The role of GABA in the regulation of the dopamine/tyrosine hydroxylase-containing neurons of the rat retina. Brain Res 214, 335-347.

31. Deans, M.R., Volgyi, B., Goodenough, D.A., Bloomfield, S.A., and Paul, D.L. (2002). Connexin36 is essential for transmission of rod-mediated visual signals in the mammalian retina. Neuron $36,703-712$.

32. Volgyi, B., Deans, M.R., Paul, D.L., and Bloomfield, S.A. (2004). Convergence and segregation of the multiple rod pathways in mammalian retina. J Neurosci 24, 11182-11192.

33. Puopolo, M., Hochstetler, S.E., Gustincich, S., Wightman, R.M., and Raviola, E. (2001). Extrasynaptic release of dopamine in a retinal neuron: activity dependence and transmitter modulation. Neuron 30, 211-225.

34. Jin, N.G., Chuang, A.Z., Masson, P.J., and Ribelayga, C.P. (2015). Rod electrical coupling is controlled by a circadian clock and dopamine in mouse retina. J Physiol 593, 1597-1631.

35. Xin, D., and Bloomfield, S.A. (1999). Dark- and light-induced changes in coupling between horizontal cells in mammalian retina. J Comp Neurol 405, 75-87.

36. Bloomfield, S.A., and Volgyi, B. (2004). Function and plasticity of homologous coupling between All amacrine cells. Vision Res 44, 3297-3306.

37. Hampson, E.C., Vaney, D.I., and Weiler, R. (1992). Dopaminergic modulation of gap junction permeability between amacrine cells in mammalian retina. J Neurosci 12 , 4911-4922.

38. Allen, A.E., and Lucas, R.J. (2016). Using Silent Substitution to Track the Mesopic Transition From Rod- to Cone-Based Vision in Mice. Invest Ophthalmol Vis Sci 57, 276-287.

39. Soucy, E., Wang, Y., Nirenberg, S., Nathans, J., and Meister, M. (1998). A novel signaling pathway from rod photoreceptors to ganglion cells in mammalian retina. Neuron 21, 481-493.

40. Herrmann, R., Heflin, S.J., Hammond, T., Lee, B., Wang, J., Gainetdinov, R.R., Caron, M.G., Eggers, E.D., Frishman, L.J., McCall, M.A., et al. (2011). Rod vision is controlled by dopamine-dependent sensitization of rod bipolar cells by GABA. Neuron 72, 101110.

41. Threlfell, S., Lalic, T., Platt, N.J., Jennings, K.A., Deisseroth, K., and Cragg, S.J. (2012). Striatal dopamine release is triggered by synchronized activity in cholinergic interneurons. Neuron 75, 58-64.

42. Kolb, H., Cuenca, N., Wang, H.H., and Dekorver, L. (1990). The Synaptic Organization of the Dopaminergic Amacrine Cell in the Cat Retina. Journal of Neurocytology 19, 343-366.

43. Witkovsky, P., Gabriel, R., and Krizaj, D. (2008). Anatomical and neurochemical characterization of dopaminergic interplexiform processes in mouse and rat retinas. J Comp Neurol 510, 158-174.

44. Grimes, W.N., Zhang, J., Graydon, C.W., Kachar, B., and Diamond, J.S. (2010). Retinal parallel processors: more than 100 independent microcircuits operate within a single interneuron. Neuron 65, 873-885.

45. Marc, R.E., Jones, B.W., Anderson, J.R., Kinard, K., Marshak, D.W., Wilson, J.H., Wensel, T., and Lucas, R.J. (2007). Neural reprogramming in retinal degeneration. Invest Ophthalmol Vis Sci 48, 3364-3371. 
46. Cameron, M.A., Suaning, G.J., Lovell, N.H., and Morley, J.W. (2013). Electrical stimulation of inner retinal neurons in wild-type and retinally degenerate $(\mathrm{rd} / \mathrm{rd})$ mice. PLoS ONE 8, e68882.

47. Ho, T., Jobling, A.I., Greferath, U., Chuang, T., Ramesh, A., Fletcher, E.L., and Vessey, K.A. (2015). Vesicular expression and release of ATP from dopaminergic neurons of the mouse retina and midbrain. Front Cell Neurosci 9, 389.

48. Hirasawa, H., Contini, M., and Raviola, E. (2015). Extrasynaptic release of GABA and dopamine by retinal dopaminergic neurons. Philosophical transactions of the Royal Society of London 370.

49. Zhou, Y., Tencerova, B., Hartveit, E., and Veruki, M.L. (2016). Functional NMDA receptors are expressed by both All and A17 amacrine cells in the rod pathway of the mammalian retina. J Neurophysiol 115, 389-403.

50. Sun, F., Zeng, J., Jing, M., Zhou, J., Feng, J., Owen, S.F., Luo, Y., Li, F., Wang, H., Yamaguchi, T., et al. (2018). A Genetically Encoded Fluorescent Sensor Enables Rapid and Specific Detection of Dopamine in Flies, Fish, and Mice. Cell 174, 481-496 e419.

51. Patriarchi, T., Cho, J.R., Merten, K., Howe, M.W., Marley, A., Xiong, W.H., Folk, R.W., Broussard, G.J., Liang, R., Jang, M.J., et al. (2018). Ultrafast neuronal imaging of dopamine dynamics with designed genetically encoded sensors. Science 360.

52. Govardovskii, V.I., Fyhrquist, N., Reuter, T., Kuzmin, D.G., and Donner, K. (2000). In search of the visual pigment template. Vis Neurosci 17, 509-528.

53. Field, G.D., and Rieke, F. (2002). Mechanisms regulating variability of the single photon responses of mammalian rod photoreceptors. Neuron 35, 733-747.

54. Jacobs, G.H., and Williams, G.A. (2007). Contributions of the mouse UV photopigment to the ERG and to vision. Doc Ophthalmol 115, 137-144.

55. Lyubarsky, A.L., Daniele, L.L., and Pugh, E.N., Jr. (2004). From candelas to photoisomerizations in the mouse eye by rhodopsin bleaching in situ and the lightrearing dependence of the major components of the mouse ERG. Vision Res 44, 3235-3251.

56. Milosavljevic, N., Cehajic-Kapetanovic, J., Procyk, C.A., and Lucas, R.J. (2016). Chemogenetic Activation of Melanopsin Retinal Ganglion Cells Induces Signatures of Arousal and/or Anxiety in Mice. Curr Biol 26, 2358-2363.

57. Peachey, N.S., Alexander, K.R., and Fishman, G.A. (1989). The luminance-response function of the dark-adapted human electroretinogram. Vision Res 29, 263-270. 


\section{Figure legends}

\section{Figure 1. Light-induced dopamine release is high-threshold.}

A) Two complementary methods were used to measure dopamine release. In vivo: anaesthetised mice were light pulsed, retinae extracted and DOPAC:DA ratio quantified. Ex vivo: Eyes were removed from the animal, cornea, iris and lens removed and the eye submerged in artificial cerebrospinal fluid (aCSF). Eyes were light pulsed in plastic tubes and the supernatant aCSF was analysed for dopamine content (maximum duration of pulse was $15 \mathrm{~min})$.

B) Significant light-induced dopamine turnover in vivo occurs only above 5 Log R* $\mathrm{rod}^{-1} \mathrm{~s}^{-1}$ compared to dark-adapted mice dissected under dim red light $\left(0.017 \log R^{*} \operatorname{rod}^{-1} \mathrm{~s}^{-1},>650\right.$ $\mathrm{nm}$ ). One-way ANOVA with Dunnett's multiple comparison test ( $n \geq 4$ each point; all white pulses $1 \mathrm{hr}$ ).

C) Light-induced dopamine release ex vivo showed the same threshold as in vivo. One-way ANOVA with Dunnett's multiple comparison test ( $n \geq 5$ each point; all white pulses $15 \mathrm{~min}$ ).

D) After normalisation ( $\left.\mathrm{R} / \mathrm{R}_{\max }\right)$ in vivo and ex vivo data can be fit with the same curve (extra sum-of-squares $F$ test, $p=0.53, F=0.79$ ). ERG $b$-wave amplitude in response to the same light stimulus (but $10 \mathrm{~ms}$ flashes) is plotted for reference ( $n=6$ each point).

E) Dopamine turnover is significantly greater in dark-adapted mice dissected under infrared light (-2.5 Log $\mathrm{R}^{*} \operatorname{rod}^{-1} \mathrm{~s}^{-1}$, >800 $\mathrm{nm}$ ) compared to those dissected under dim red light $(0.017$ Log $R^{*}$ rod $\left.^{-1} \mathrm{~s}^{-1},>650 \mathrm{~nm}\right)$. Unpaired Student's t-test ${ }^{* * *} p<0.0001$ ( $\mathrm{n} \geq 6$; animals exposed to light for $<1 \mathrm{~min}$ ).

F) No statistical differences are observed between equivalent irradiance points at subjective midday (CT6; $n \geq 4$ each point) and subjective midnight (CT18; $n \geq 3$ each point), one curve fits all data sets (extra sum-of-squares $F$ test, $p=0.84 ; F=0.34$; all white pulses $1 \mathrm{hr}$ ). Mean \pm SEM for all data points presented.

Figure 2. Cone input is not required for light-induced dopamine release.

A) Gnat2 ${ }^{A 518 G}$ ( $n \geq 9$ each point) mice lacking cone function show an indistinguishable dopamine irradiance response to wild-type mice ( $n \geq 4$ each point), one curve fits all data sets (extra sum-of-squares $\mathrm{F}$ test, $p=0.7 ; F=0.54$; all white pulses $1 \mathrm{hr}$ ).

B) Dopamine turnover is significantly greater in dark-adapted Gnat2 ${ }^{A 518 G}$ mice dissected under infrared light $\left(-2.5 \mathrm{Log} \mathrm{R}^{*} \operatorname{rod}^{-1} \mathrm{~s}^{-1},>800 \mathrm{~nm}\right)$ compared to those dissected under dim red light ( 0.017 Log $\mathrm{R}^{*}$ rod $\left.^{-1} \mathrm{~s}^{-1},>650 \mathrm{~nm}\right)$. Unpaired Student's t-test $* * p<0.01(\mathrm{n} \geq 6$; animals exposed to light for $<1 \mathrm{~min}$ ).

C) No statistical differences are observed between equivalent irradiance points at subjective midday (CT6; $n \geq 9$ each) and subjective midnight (CT18; $n \geq 3$ each point), one curve fits all data sets (extra sum-of-squares $F$ test, $p=0.93 ; F=0.2$; all white pulses $1 \mathrm{hr}$ ).

D-F) Representative light-adapted electroretinograms (ERGs) recorded in Gnat2 ${ }^{A 518 G}$ and wild-type mice in response to 0.99 Michaelson contrast at two different background luminances reveal that measurable rod-driven responses can be recorded in Gnat $2^{A 518 G}$ with a background below (E) but not above (F) the dopamine release threshold. Animals were adapted to the background for 20 min prior to recording.

G) Opn $1^{m w R}$ mice do not exhibit significant light-induced dopamine release in response a redlight pulse (624 nm; $15 \mathrm{~min}$ ) designed to activate L-cones above the dopamine release threshold but not rods or Opn4. Normal amplitude dopamine release still occurs under bright 
white light (6.8 Log $\left.\mathrm{R}^{*} \operatorname{rod}^{-1} \mathrm{~s}^{-1} ; 15 \mathrm{~min}\right)$. One-way ANOVA Dunnett's multiple comparison test $\left({ }^{*} p<0.05, n \geq 5\right)$.

H) When red (624 nm; $15 \mathrm{~min}$ ) and blue (434 nm; $15 \mathrm{~min}$ ) light-pulses are matched for rod sensitivity in wild-type animals both cause significant dopamine release compared to dim-red light. One-way ANOVA Dunnett's multiple comparison test ( $* * * p<0.001, n=5)$.

I) No light-induced dopamine release occurs in wild-type mice at 4.6 Log $R^{*}$ rod $^{-1} \mathrm{~s}^{-1}$ under either continuous or flickering ( $0.5 \mathrm{sec} O \mathrm{~N}, 1.5 \mathrm{sec}$ OFF; total $15 \mathrm{~min}$ ) light. One-way ANOVA $(p>0.05, n \geq 4$ each). All data points mean \pm SEM. See Figure S1 for more information on the red and blue pulses.

Figure 3. mRGCs are not necessary or sufficient to drive light-induced dopamine release.

A) $r d / r d$ mice lacking rods and cones do not show light-induced dopamine release at any given light intensity. One-way ANOVA ( $p>0.05, n \geq 12$ each).

B) Mice lacking melanopsin (Opn $4^{\mathrm{Cre} / \mathrm{Cre}}$ ) show an indistinguishable dopamine irradiance response to wild-types ( $n \geq 4$ each), one curve fits both data sets (extra sum-of-squares $F$ test, $p=0.88 ; F=0.3$ ).

C) Dopamine turnover is significantly greater under infrared light $\left(-2.5 \operatorname{Log~R}^{*} \operatorname{rod}^{-1} \mathrm{~s}^{-1},>800\right.$ $\mathrm{nm}$ ) compared to dim red light (0.017 Log $\left.\mathrm{R}^{*} \operatorname{rod}^{-1} \mathrm{~s}^{-1},>650 \mathrm{~nm}\right)$ in Opn4 Cre/Cre, but not $r d / r d$ mice. Unpaired Student's t-test ( ${ }^{* *} p<0.01$, mean \pm SEM, $n \geq 4$ each).

D) $r d / r d$ retinae release dopamine in a high-potassium media $(15 \mathrm{mM})$ in both dim red and light conditions. One-way ANOVA with Tukey's multiple comparison tests ( ${ }^{*} p<0.05$, mean \pm SEM, $n \geq 5$ each).

E) Injection of CNO (5 mg/kg) caused activation of mRGCs in Opn $4^{\text {Cre/+ }} \mathrm{hM} 3 \mathrm{Dq}$-infected mice. Top: representative image of infected eye before, and 10 min after, i.p. injection of CNO (contralateral uninfected eye showed similar constriction). Bottom: immunohistochemistry of a vertical retinal section showing co-localisation of c-fos (green, marker for neuronal activation) and mCherry (red, transfected mRGCs; scale bar $30 \mu \mathrm{m}$ ).

G) Activation of mRGCs by CNO does not cause increased dopamine turnover in the hM3Dqinfected eye of Opn $4^{\mathrm{Cre} /+}$ mice. Paired two-tailed t-test, left vs right eye ( $p=0.6$, mean $\pm \mathrm{SEM}$, $n=5$ each). $O p n 4^{+/+}$littermates show normal light-induced dopamine release, unpaired Student's t-test, $* * * p<0.001$.

$\mathrm{H})$ Immunohistochemistry reveals that dopaminergic amacrine cells (labelled with TH in red) express c-fos (green) in wild-type mice in response to a 90 min light-pulse, but not in the hM3Dq-infected eye of Opn4 $4^{\text {Cre/t }}$ mice 90 min after i.p. CNO injection. No c-fos was observed in $\operatorname{dim} \mathrm{red} /$ control conditions (scale bar $30 \mu \mathrm{m}$ ). See Figure S2 for more details on mRGC activation by CNO.

\section{Figure 4. Effect of pharmacological blockers on dopamine release.}

A) Glycine receptor antagonist strychnine significantly reduces dopamine release in dark and has no effect on light-pulsed retinae when compared to control $(p<0.05)$. GABA $A / C$ receptor antagonist picrotoxin significantly increases dopamine turnover in the dark up to control lightpulsed levels, without additional effect to treated light-pulsed retinae (one-way ANOVA with Tukey's multiple comparisons ( $p<0.001 ; n=4$ each). \# denotes significant difference to equivalent control conditions (dotted lines).

B) Light-induced dopamine release is abolished by the mGluR6 receptor agonist L-AP4, and dopamine release is significantly decreased in both dark-adapted and light-pulsed conditions compared to controls $(p<0.05)$. AMPA/kainite receptor antagonist CNQX significantly 
prevents light-induced dopamine release $(p<0.001)$ without any effect on dark-adapted retinae. NMDA receptor antagonist AP5 significantly reduces light- induced dopamine release $(p<0.001)$, but a difference between dim-red and light treated is maintained $\left({ }^{*} p<0.05\right)$. Oneway ANOVA with Tukey's multiple comparisons between treated and untreated, \# denotes significant difference to equivalent control conditions (dotted lines). Unpaired Student's ttest within treatment groups ( $n=4$ each).

C) Gap-junction blocker meclofenamic acid (MFA) significantly reduces, but does not block, light induced dopamine release in both wild-type and cone-inactivated Gnat $2^{A 518 G}$ mice. Oneway ANOVA with Tukey's multiple comparisons between treated and untreated; unpaired Student's t-test within treatment groups $\left({ }^{* *} p<0.01,{ }^{* *} p<0.001\right.$; \# denotes significant difference to equivalent control conditions; $n \geq 4$ each; mean \pm SEM for all data points).

\section{Figure 5: Dopamine release requires spiking and synaptic input.}

A) Block of voltage-gated sodium channels by tetrodotoxin (TTX) significantly prevents lightinduced dopamine release in wild-type mice. One-way ANOVA with Tukey's multiple comparisons between treated and untreated; unpaired Student's t-test within treatment groups ( $* p<0.05$, mean \pm SEM, $n=4$ each).

B) Media containing high concentration of potassium ( $15 \mathrm{mM} \mathrm{KCl}$ ) significantly enhances lightinduced dopamine release $\left({ }^{*} p<0.05\right)$ but has no effect under dim red light conditions. Oneway ANOVA with Tukey's multiple comparisons between treated and untreated; unpaired Student's t-test within treatment groups (mean \pm SEM, $n=4$ each).

C) Dopamine turnover is increased in $r d / r d$ mice in $\mathrm{Mg}^{2+}$-free media under both dim red and bright light conditions. One-way ANOVA with Tukey's multiple comparisons between treated and untreated ( ${ }^{* *} p<0.01$, mean $\pm \mathrm{SEM}, n=4$ each). All $y$-axes same units.

\section{Figure 6: Duration response characteristics of dopamine release}

A) Significant increase in light-induced dopamine turnover in vivo occurs after $30 \mathrm{sec}$ of light exposure (6.8 $\mathrm{Log}^{*}$ rod $^{-1} \mathrm{~s}^{-1}$ ) compared to dark-adapted mice dissected under dim red light. This difference is maintained as long as tested $(60 \mathrm{~min})$. When lights are turned off from a light-adapted retina, a significant decrease in dopamine release results after $10 \mathrm{~min}$. The decrease continues for $60 \mathrm{~min}$ until overnight dark-adapted levels are reached. One-way ANOVA with Dunnett's multiple comparison test $(p<0.05, n \geq 4$ each point except $t=65,90$, 120 min $\mathrm{n}=2$ ).

B) Ex vivo dopamine release displays a gradual increase over $15 \mathrm{~min}$ that can be fit with a sigmoidal curve (dotted line; $n=2-6$ each point). In vivo data is repeated for comparison (black line).

C-D) Matlab Simulink model - (i) Light input to the system - LIGHT ON intensity is an arbitrary value (0.8) chosen to more closely replicate the empirical response, LIGHT OFF has a value of 0 . The primary dopamine release response is switched between alternate pathways depending on the light condition. (ii) Dopamine release output of the model (iii) Negative feedback to light input - Light input to these pathways is reduced by negative only delayed feedback system which has dopamine release as an input. (iv) Light mediated direct inhibition of dopamine release.

Figure 7: Mechanism of dopamine release in the mouse retina [in discussion]

Suggested model to describe dopamine release in the mouse retina. Left: in the dark no light input to dopaminergic varicosities means dopamine release is not inhibited resulting in a high 
basal release. Middle: scotopic/mesopic rod activation drives GABAergic inhibition, and a small excitatory glutamatergic input only to synaptic AMPA/kainite receptors causing reduced basal dopamine release. Right: activation by bright light also drives GABAergic inhibition but activates extra-synaptic NMDA receptors via glutamate spill over resulting in more excitation than inhibition causing a large release of dopamine.

Figure S1: In vivo and ex vivo dopamine release comparison for wild-type, Gnat2 ${ }^{A 518 G}$ and Opn4 ${ }^{\mathrm{Cre} / \mathrm{Cre}}$ animals. Related to Figures 1,2 \& 3.

A) In vivo DOPAC:DA ratio was normalised $\left(R / R_{\max }\right)$ to reveal that one curve fits release profiles for wild-type, Gnat2 ${ }^{A 518 G}$ and $O p n 4^{C r e / C r e}$ animals (extra sum-of-squares $F$ test, $p=0.99 ; F=$ $0.2)$.

B) Ex vivo dopamine release ( $\mathrm{pg} /$ retina) was normalised $\left(\mathrm{R} / \mathrm{R}_{\max }\right)$ to reveal that one curve fits release profiles for wild-type, Gnat $2^{A 518 G}$ and $O p n 4^{C r e / C r e}$ animals (extra sum-of-squares F test, $p=0.3 ; F=1.2) . E C_{50}$ of the fitted curves for each genotype was not significantly different between in vivo and ex vivo (paired Student's t-test $p>0.05 ; n \geq 3$ each point).

\section{Figure S2: Red and blue stimuli. Related to Figure 2.}

A) Top: dotted red line - red light pulse ( $624 \mathrm{~nm}$; $15 \mathrm{~min}$ ) used in Figure 2G. Bottom: red solid and blue dotted lines - rod matched stimuli used in Figure $2 \mathrm{H}$.

$\mathrm{B}-\mathrm{F})$ Theoretical dopamine release curves in response to white light plotted against effective $\log$ photons $\mathrm{cm}^{-2} \mathrm{~s}^{-1}$ assuming this response is driven by $\mathrm{B}$ - rods $(498 \mathrm{~nm}), \mathrm{C}-\mathrm{M}$-cones $(508$ $\mathrm{nm}), \mathrm{D}-\mathrm{L}$-cones $(556 \mathrm{~nm}), \mathrm{E}-\mathrm{UV}$-cones $\left(360 \mathrm{~nm}\right.$ ) or F - melanopsin (480 $\left.\mathrm{nm} \lambda_{\max }\right)$. Red and blue pulse effective log photons $\mathrm{cm}^{-2} \mathrm{~s}^{-1}$ for each photoreceptor is plotted on each individual graph. Inserts: B, ERG response in a wild-type mouse to a dim version of the rod matched red and blue stimuli (10 rod effective log photons $\mathrm{cm}^{-2} \mathrm{~s}^{-1}$; below cone threshold; scale bar 200 $\mathrm{ms}, 50 \mu \mathrm{V})$. D, ERG flicker $(40 \mathrm{~Hz})$ response of wild-type (green) and Opn1 ${ }^{\mathrm{mwR}}$ (red) mice to the red light pulse (dotted red; scale bar $100 \mathrm{~ms}, 10 \mu \mathrm{V}$ ).

\section{Figure S3: Activation of mRGCs in Opn ${ }^{\mathrm{Cre} /+} \mathrm{hM} 3 \mathrm{Dq}$-infected animals with clozapine $\mathrm{N}$-oxide} (CNO). Related to Figure 3.

A) Intraperitoneal injection (i.p.) of CNO causes prolonged pupil constriction under infrared illumination (>950 $\mathrm{nm}$ ) that begins to subside $4 \mathrm{hrs}$ after injection.

B) hM3Dq-expressing cells can be visualized by mCherry (red) expression. Yellow/green cell shows both mCherry expression and biocytin (green) following patch-clamp recording (scale bar $30 \mu \mathrm{m})$.

C) Current-clamp recording from yellow/green cell in B shows that a local puff of $10 \mu \mathrm{M}$ CNO causes rapid depolarisation and spiking that decreases following the puff.

D) Slow bath application of $10 \mathrm{nM}$ CNO initially causes neuronal silencing for $~ 2.5 \mathrm{~min}$, followed by a large increase in firing rate that was maintained for the recording ( $>60 \mathrm{~min}$; trace truncated). 
A

(anaesthetised)

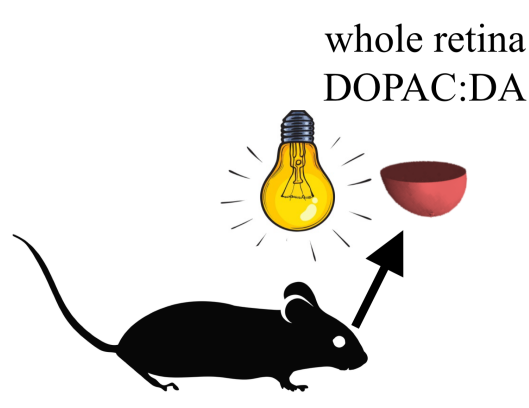

ex vivo

(RPE attached)

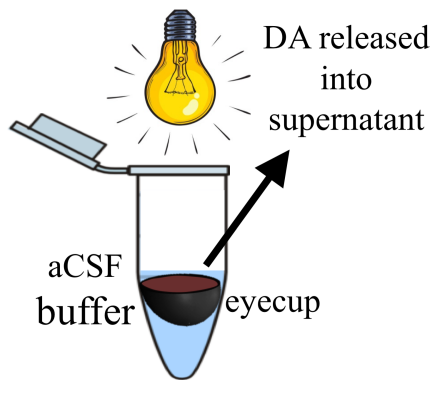

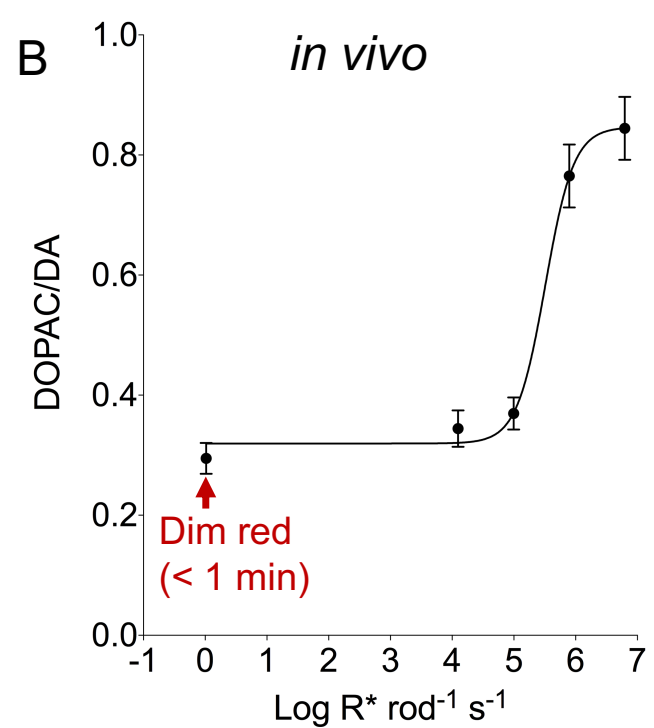
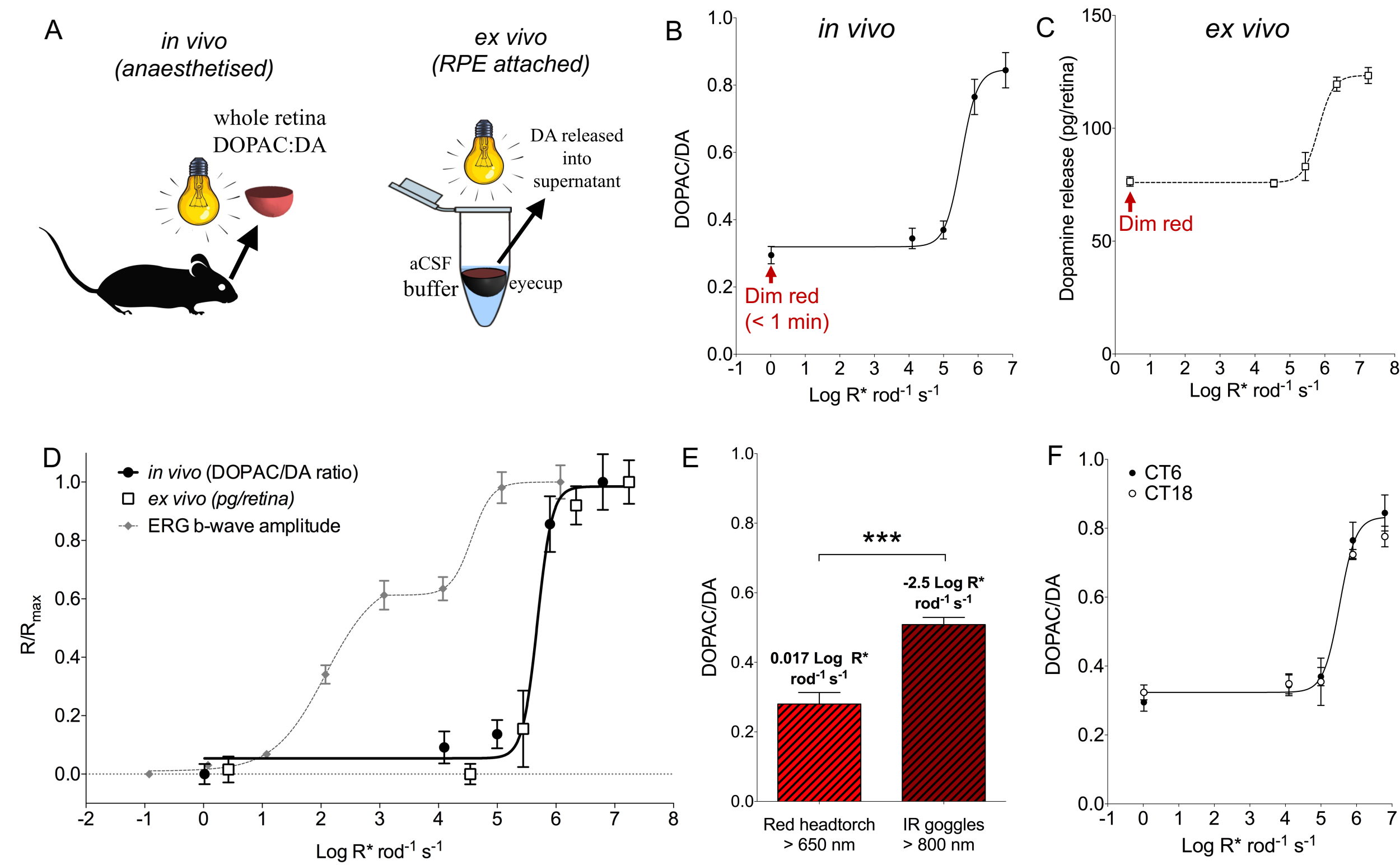

Figure 1. Light-induced dopamine release is high-threshold.

A) Two complementary methods were used to measure dopamine release. In vivo: anaesthetised mice were light pulsed, retinae extracted and DOPAC:DA ratio quantified. Ex vivo: Eyes were removed from the animal, cornea, iris and lens removed and the eye submerged in artificial cerebrospinal fluid (aCSF). Eyes were light pulsed in plastic tubes and the supernatant aCSF was analysed for dopamine content (maximum duration of pulse was $15 \mathrm{~min}$ ).

B) Significant light-induced dopamine turnover in vivo occurs only above 5 Log $\mathrm{R}^{*}$ rod $^{-1} \mathrm{~s}^{-1}$ compared to dark-adapted mice dissected under dim red light ( $\left.0.017 \mathrm{Log} \mathrm{R}^{*} \operatorname{rod}^{-1} \mathrm{~s}^{-1},>650 \mathrm{~nm}\right)$. One-way ANOVA with Dunnett's multiple comparison test ( $n \geq 4$ each point; all white pulses $1 \mathrm{hr}$ ).

C) Light-induced dopamine release ex vivo showed the same threshold as in vivo. One-way ANOVA with Dunnett's multiple comparison test ( $n \geq$ 5 each point; all white pulses $15 \mathrm{~min})$.

D) After normalisation $\left(R / R_{\max }\right)$ in vivo and ex vivo data can be fit with the same curve (extra sum-of-squares $F$ test, $\left.p=0.53, F=0.79\right)$. ERG $b$ wave amplitude in response to the same light stimulus (but $10 \mathrm{~ms}$ flashes) is plotted for reference ( $\mathrm{n}=6$ each point).

E) Dopamine turnover is significantly greater in dark-adapted mice dissected under infrared light $\left(-2.5 \operatorname{Log~R}^{*} \operatorname{rod}^{-1} \mathrm{~s}^{-1},>800 \mathrm{~nm}\right) \operatorname{compared}$ to those dissected under dim red light $\left(0.017 \operatorname{Log~R}^{*} \operatorname{rod}^{-1} \mathrm{~s}^{-1},>650 \mathrm{~nm}\right)$. Unpaired Student's t-test $* * * p<0.0001$ ( $\mathrm{n} \geq 6$; animals exposed to light for $<1 \mathrm{~min})$.

F) No statistical differences are observed between equivalent irradiance points at subjective midday (CT6; $n \geq 4$ each point) and subjective midnight (CT18; $n \geq 3$ each point), one curve fits all data sets (extra sum-of-squares $F$ test, $p=0.84 ; F=0.34$; all white pulses $1 \mathrm{hr}$ ). Mean $\pm \mathrm{SEM}$ for all data points presented. 

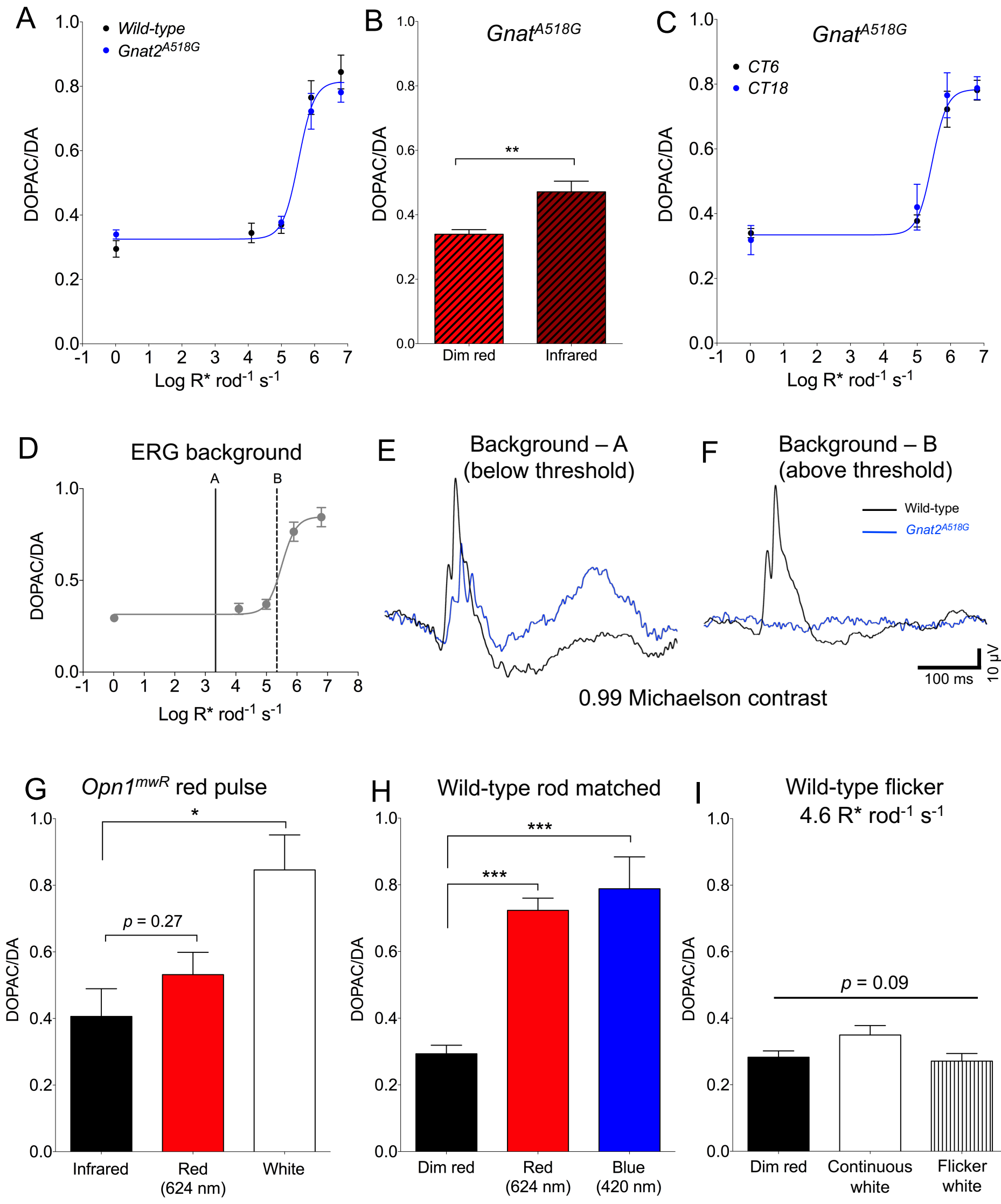

Figure 2. Cone input is not required for light-induced dopamine release.

A) Gnat2 ${ }^{A 518 G}$ ( $n \geq 9$ each point) mice lacking cone function show an indistinguishable dopamine irradiance response to wild-type mice ( $n \geq 4$ each point), one curve fits all data sets (extra sum-of-squares $\mathrm{F}$ test, $p=$ $0.7 ; F=0.54$; all white pulses $1 \mathrm{hr}$ ).

B) Dopamine turnover is significantly greater in dark-adapted Gnat2 ${ }^{A 518 G}$ mice dissected under infrared light $\left(-2.5 \mathrm{Log} \mathrm{R}^{*} \operatorname{rod}^{-1} \mathrm{~s}^{-1}\right.$, >800 $\left.\mathrm{nm}\right)$ compared to those dissected under dim red light $\left(0.017 \mathrm{Log} \mathrm{R}^{*} \operatorname{rod}^{-1} \mathrm{~s}^{-}\right.$ 1 , >650 nm). Unpaired Student's t-test $* * p<0.01$ ( $\mathrm{n} \geq 6$; animals exposed to light for $<1 \mathrm{~min}$ ).

C) No statistical differences are observed between equivalent irradiance points at subjective midday (CT6; $n \geq 9$ each) and subjective midnight (CT18; $n \geq 3$ each point), one curve fits all data sets (extra sum-ofsquares $\mathrm{F}$ test, $p=0.93 ; F=0.2 ;$ all white pulses $1 \mathrm{hr}$ ).

D-F) Representative light-adapted electroretinograms (ERGs) recorded in Gnat2 ${ }^{A 518 G}$ and wild-type mice in response to 0.99 Michaelson contrast at two different background luminances reveal that measurable roddriven responses can be recorded in Gnat2 ${ }^{A 518 G}$ with a background below (E) but not above (F) the dopamine release threshold. Animals were adapted to the background for $20 \mathrm{~min}$ prior to recording.

G) Opn $1^{m w R}$ mice do not exhibit significant light-induced dopamine release in response a red-light pulse (624 nm; $15 \mathrm{~min}$ ) designed to activate L-cones above the dopamine release threshold but not rods or

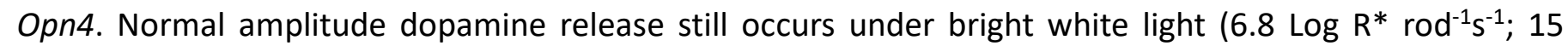
min). One-way ANOVA Dunnett's multiple comparison test $\left({ }^{*} p<0.05, n \geq 5\right)$.

$\mathrm{H})$ When red (624 nm; $15 \mathrm{~min}$ ) and blue (420 nm; $15 \mathrm{~min}$ ) light-pulses are matched for rod sensitivity in wild-type animals both cause significant dopamine release compared to dim-red light. One-way ANOVA Dunnett's multiple comparison test ( $* * * p<0.001, \mathrm{n}=5$ ).

I) No light-induced dopamine release occurs in wild-type mice at $4.6 \mathrm{Log} \mathrm{R}^{*}$ rod $^{-1} \mathrm{~s}^{-1}$ under either continuous or flickering ( $0.5 \mathrm{sec}$ ON, $1.5 \mathrm{sec}$ OFF; total $15 \mathrm{~min}$ ) light. One-way ANOVA ( $p>0.05, n \geq 4$ each). All data points mean \pm SEM. See Figure S1 for more information on the red and blue pulses. 


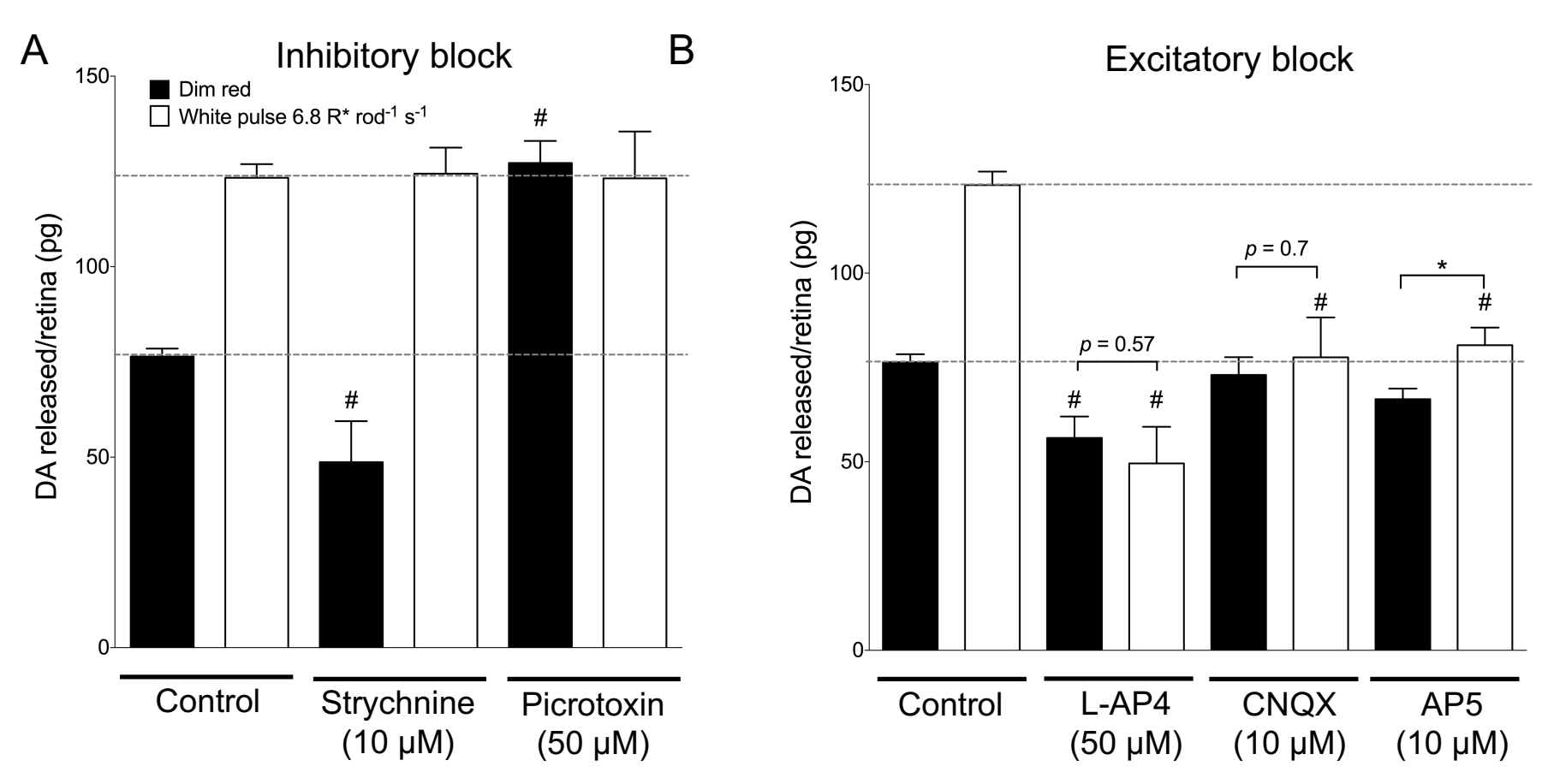

C

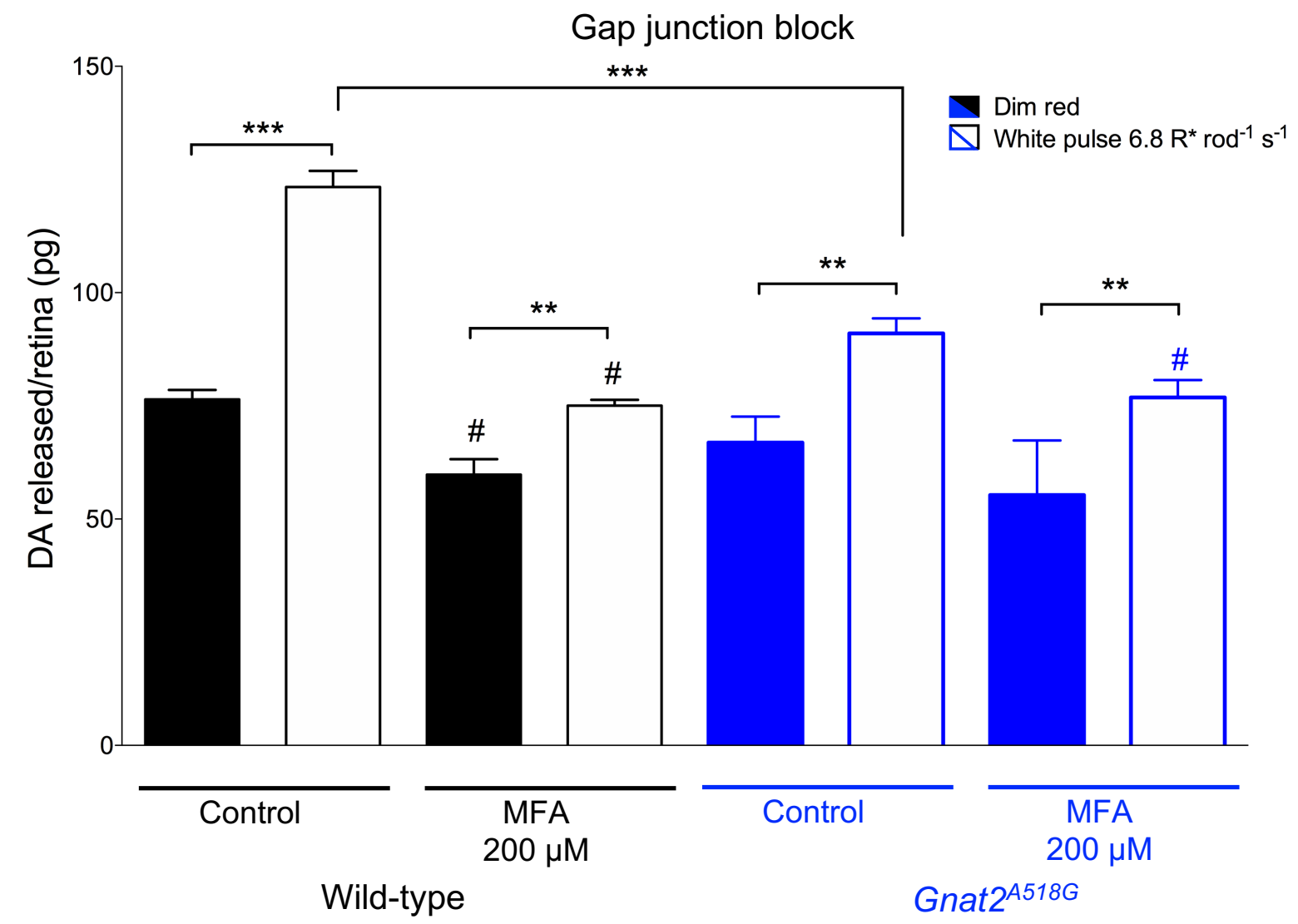

Figure 4. Effect of pharmacological blockers on dopamine release.

A) Glycine receptor antagonist strychnine significantly reduces dopamine release in dark and has no effect on light-pulsed retinae when compared to control $(p<0.05)$. GABA $A / C$ receptor antagonist picrotoxin significantly increases dopamine turnover in the dark up to control light-pulsed levels, without additional effect to treated light-pulsed retinae (oneway ANOVA with Tukey's multiple comparisons ( $p<0.001 ; n=4$ each). \# denotes significant difference to equivalent control conditions (dotted lines).

B) Light-induced dopamine release is abolished by the mGluR6 receptor agonist L-AP4, and dopamine release is significantly decreased in both dark-adapted and light-pulsed conditions compared to controls $(p<0.05)$. AMPA/kainite receptor antagonist CNQX significantly prevents light-induced dopamine release $(p<0.001)$ without any effect on dark-adapted retinae. NMDA receptor antagonist AP5 significantly reduces light- induced dopamine release $(p<0.001)$, but a difference between dim-red and light treated is maintained $(* p<0.05)$. One-way ANOVA with Tukey's multiple comparisons between treated and untreated, \# denotes significant difference to equivalent control conditions (dotted lines). Unpaired Student's t-test within treatment groups ( $n=4$ each).

C) Gap-junction blocker meclofenamic acid (MFA) significantly reduces, but does not block, light induced dopamine release in both wild-type and cone-inactivated Gnat2 ${ }^{A 518 G}$ mice. One-way ANOVA with Tukey's multiple comparisons between treated and untreated; unpaired Student's t-test within treatment groups $\left({ }^{* *} p<0.01,{ }^{* * *} p<0.001\right.$; \# denotes significant difference to equivalent control conditions; $n \geq 4$ each; mean \pm SEM for all data points). 

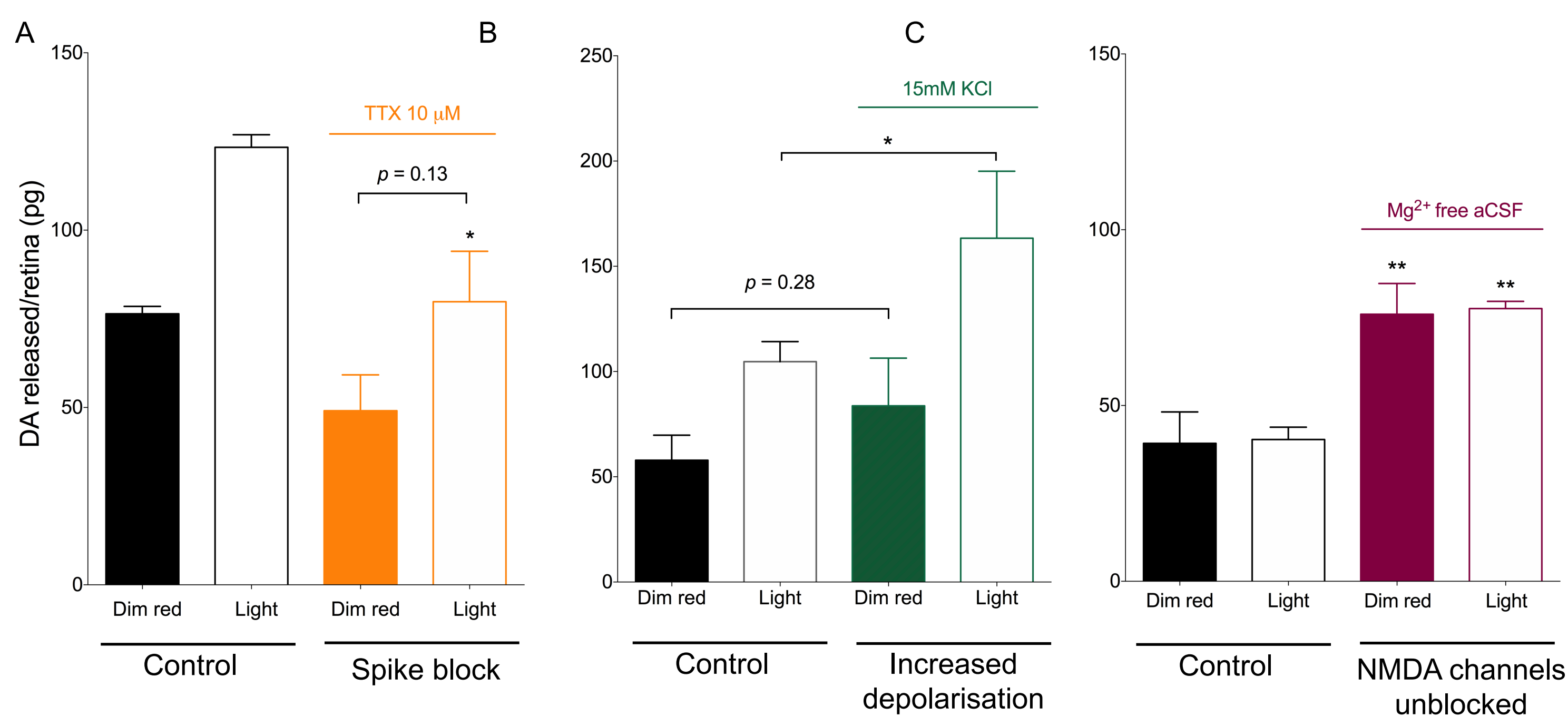

Figure 5: Dopamine release requires spiking and synaptic input.

A) Block of voltage-gated sodium channels by tetrodotoxin (TTX) significantly prevents light-induced dopamine release in wild-type mice. One-way ANOVA with Tukey's multiple comparisons between treated and untreated; unpaired Student's t-test within treatment groups $\left({ }^{*} p<0.05\right.$, mean \pm SEM, $n=4$ each).

B) Media containing high concentration of potassium $(15 \mathrm{mM} \mathrm{KCl})$ significantly enhances light-induced dopamine release $\left({ }^{*} p<0.05\right)$ but has no effect under dim red light conditions. One-way ANOVA with Tukey's multiple comparisons between treated and untreated; unpaired Student's t-test within treatment groups (mean \pm SEM, $n=4$ each).

C) Dopamine turnover is increased in $r d / r d$ mice in $\mathrm{Mg}^{2+}$-free media under both dim red and bright light conditions. One-way ANOVA with Tukey's multiple comparisons between treated and untreated ( ${ }^{*} p<0.01$, mean $\pm \mathrm{SEM}, n=4$ each). All y-axes same units. 

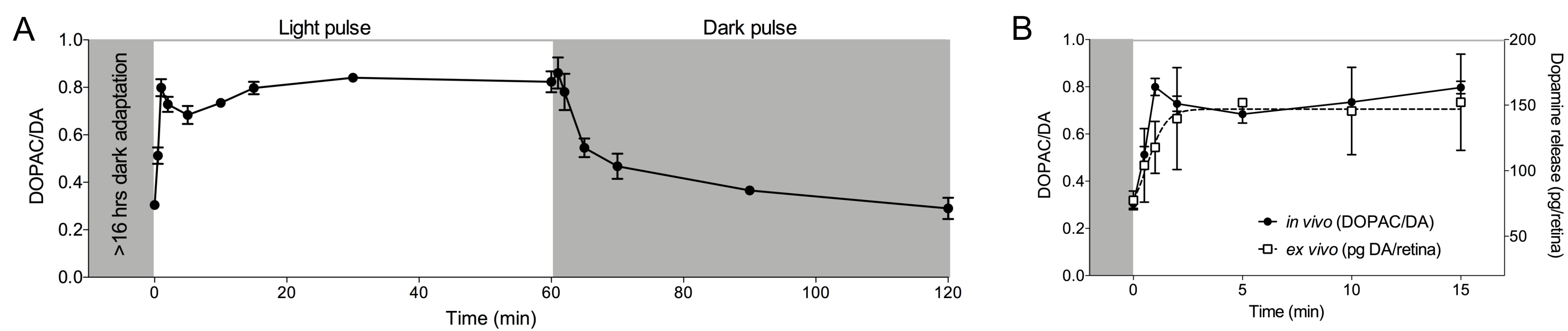

C

(i)

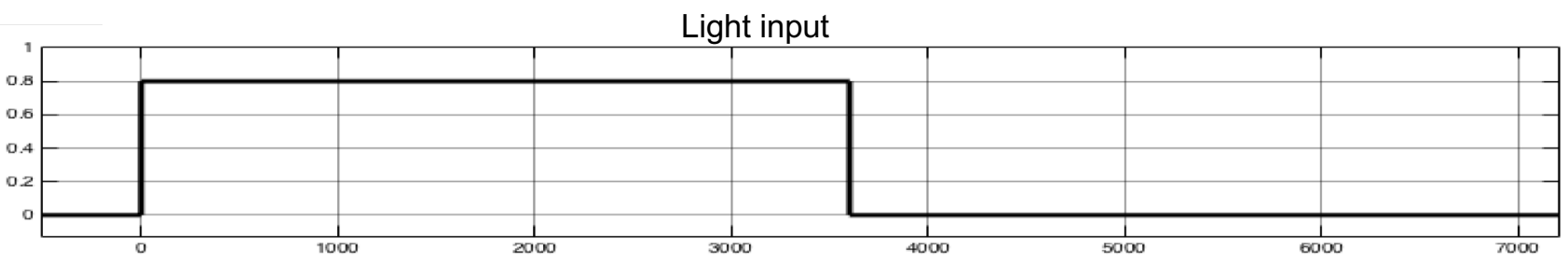

Dopamine release

(ii)

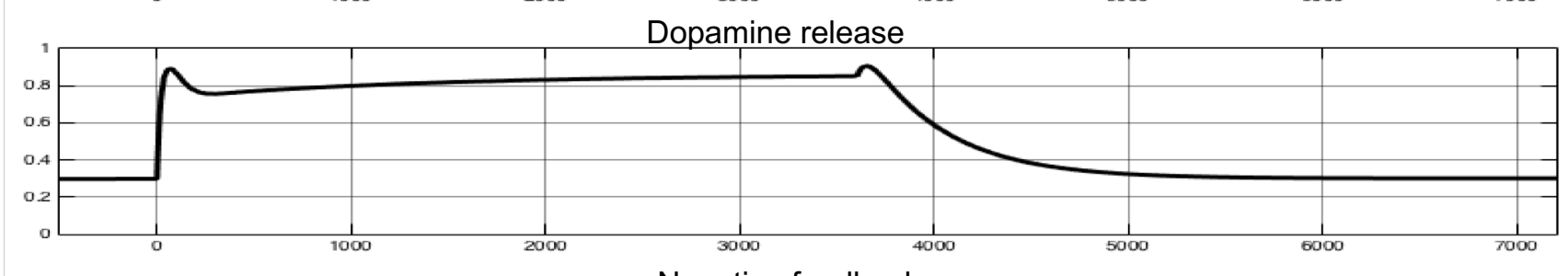

Negative feedback

(iii)

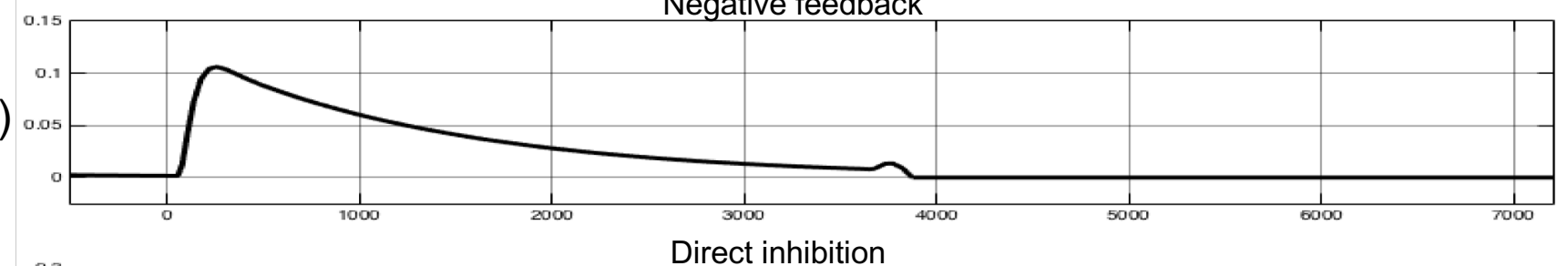

(iv)
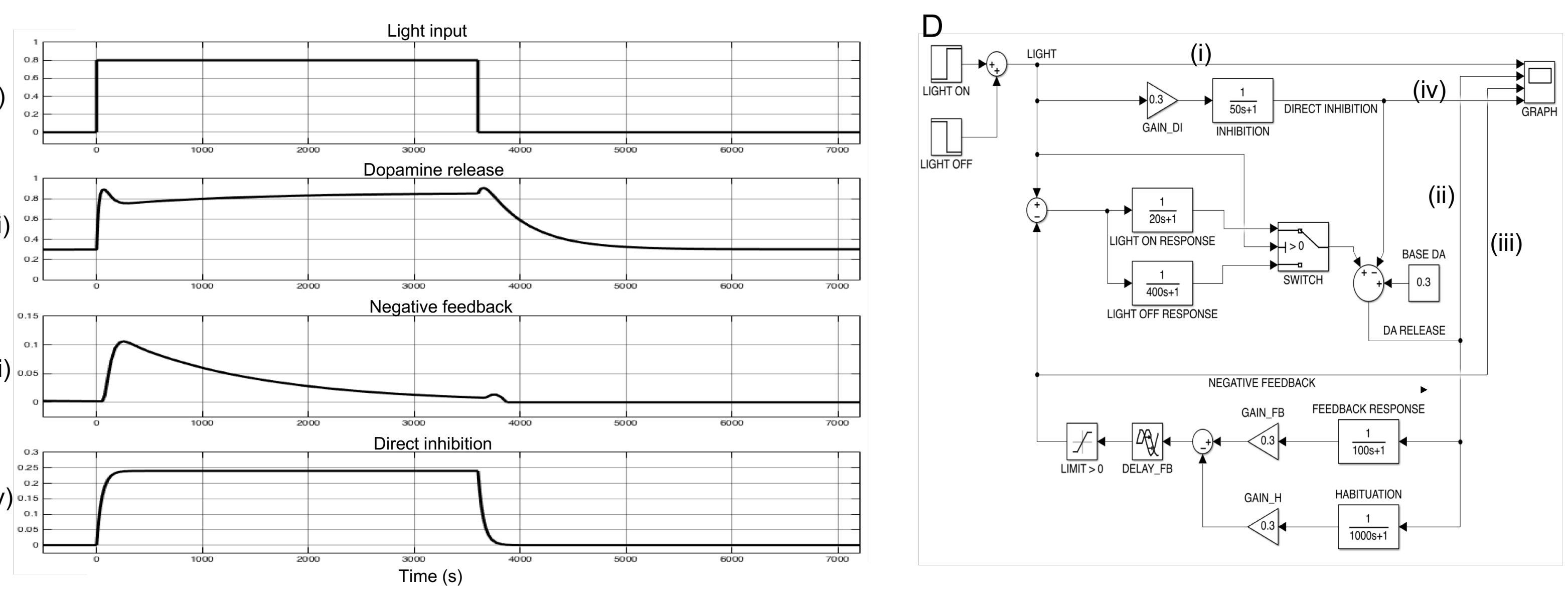

\section{Figure 6: Duration response characteristics of dopamine release}

A) Significant increase in light-induced dopamine turnover in vivo occurs after 30 sec of light exposure $\left(6.8\right.$ Log $^{*}$ rod $\left.^{-1} \mathrm{~s}^{-1}\right)$ compared to dark- $^{-1}$ adapted mice dissected under dim red light. This difference is maintained as long as tested (60 min). When lights are turned off from a lightadapted retina, a significant decrease in dopamine release results after $10 \mathrm{~min}$. The decrease continues for 60 min until overnight darkadapted levels are reached. One-way ANOVA with Dunnett's multiple comparison test $(p<0.05, n \geq 4$ each point except $t=65$, 90, 120min $\mathrm{n}=2)$.

B) Ex vivo dopamine release displays a gradual increase over 15 min that can be fit with a sigmoidal curve (dotted line; $\mathrm{n}=2-6$ each point). In vivo data is repeated for comparison (black line).

C-D) Matlab Simulink model - (i) Light input to the system - LIGHT ON intensity is an arbitrary value (0.8) chosen to more closely replicate the empirical response, LIGHT OFF has a value of 0 . The primary dopamine release response is switched between alternate pathways depending on the light condition. (ii) Dopamine release output of the model (iii) Negative feedback to light input - Light input to these pathways is reduced by negative only delayed feedback system which has dopamine release as an input. (iv) Light mediated direct inhibition of dopamine release. 


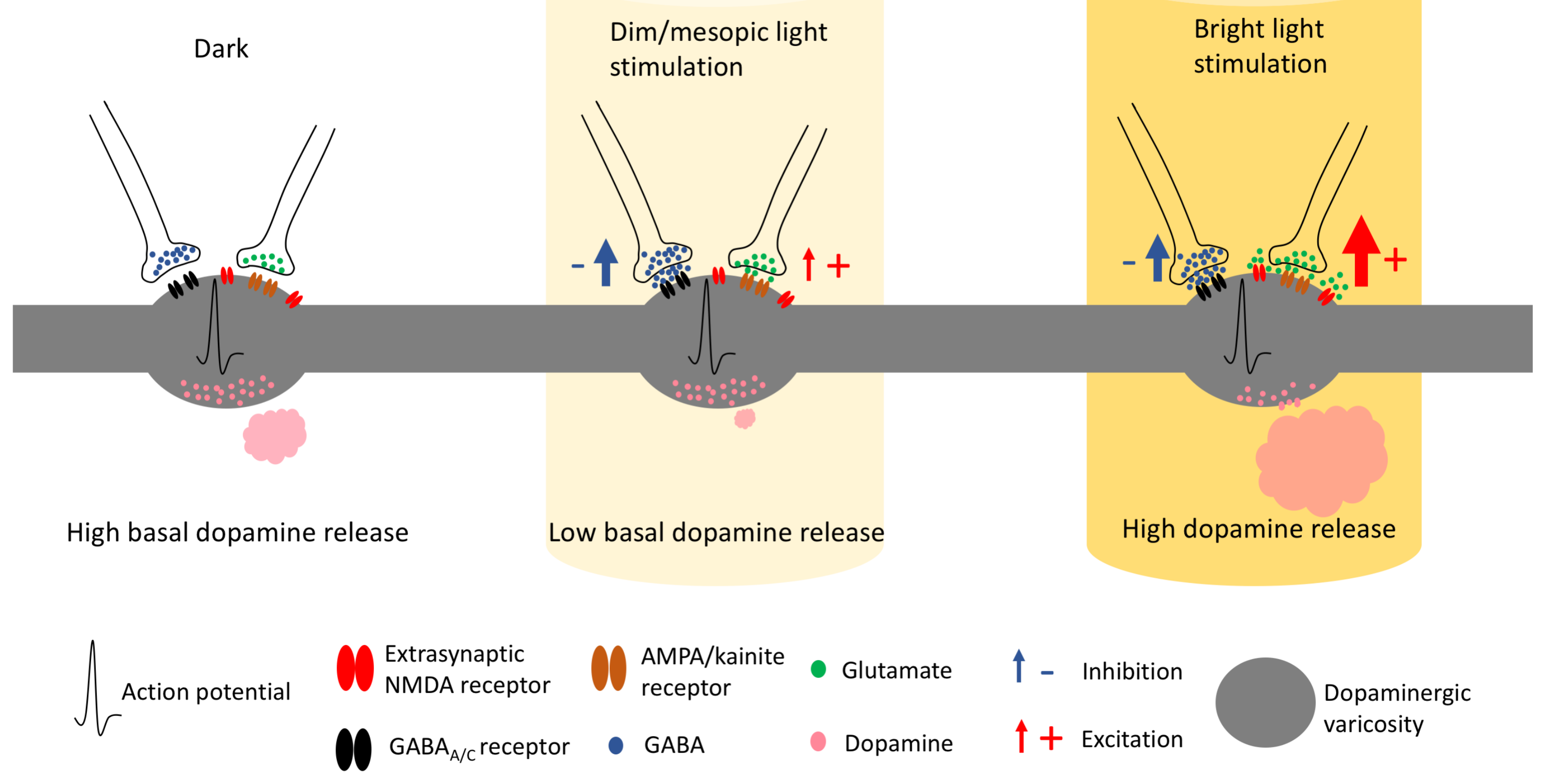

\section{Figure 7: Mechanism of dopamine release in the mouse retina [in discussion]}

Suggested model to describe dopamine release in the mouse retina. Left: in the dark no light input to dopaminergic varicosities means dopamine release is not inhibited resulting in a high basal release. Middle: scotopic/mesopic rod activation drives GABAergic inhibition, and a small excitatory glutamatergic input only to synaptic AMPA/kainite receptors causing reduced basal dopamine release. Right: activation by bright light also drives GABAergic inhibition but activates extra-synaptic NMDA receptors via glutamate spill over resulting in more excitation than inhibition causing a large release of dopamine. 

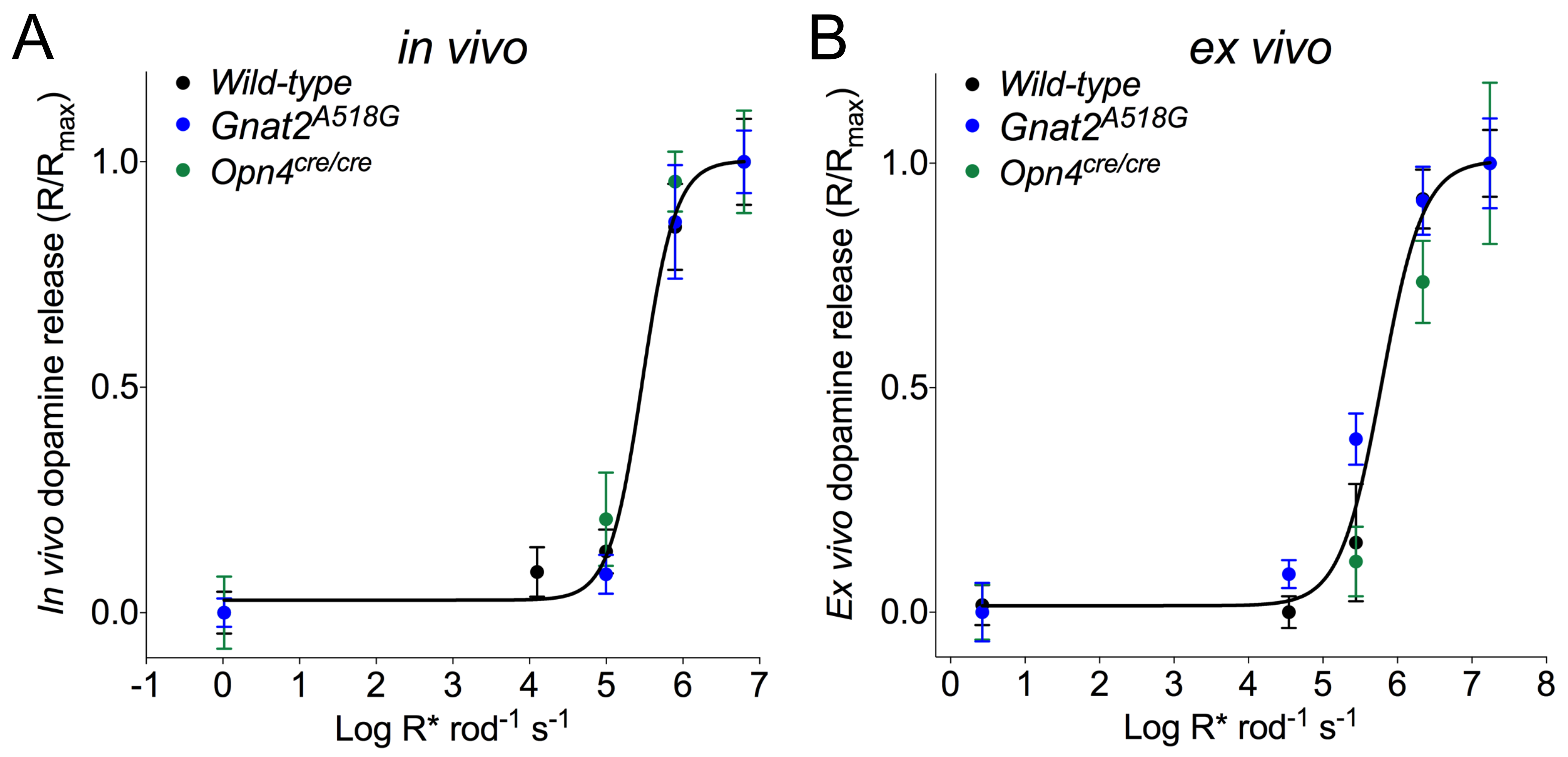

Figure S1: In vivo and ex vivo dopamine release comparison for wild-type, Gnat2 ${ }^{A 518 G}$ and

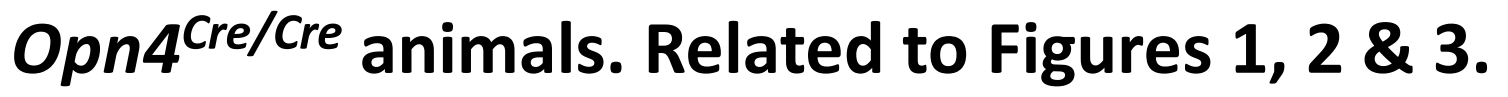

A) In vivo DOPAC:DA ratio was normalised $\left(R / R_{\max }\right)$ to reveal that one curve fits release profiles for wild-type, Gnat2 ${ }^{A 518 G}$ and Opn4 ${ }^{C r e / C r e}$ animals (extra sum-of-squares $F$ test, $p=0.99 ; F=0.2$ ).

B) Ex vivo dopamine release (pg/retina) was normalised $\left(R / R_{\max }\right)$ to reveal that one curve fits release profiles for wild-type, Gnat2 ${ }^{A 518 G}$ and $O p n 4^{C r e} /$ Cre animals (extra sum-of-squares $F$ test, $p=$ $0.3 ; F=1.2)$. $E C_{50}$ of the fitted curves for each genotype was not significantly different between in vivo and ex vivo (paired Student's t-test $p>0.05 ; n \geq 3$ each point). 
A

Red pulse (Fig 2G)

Red and blue rod matched (Fig 2H)

B

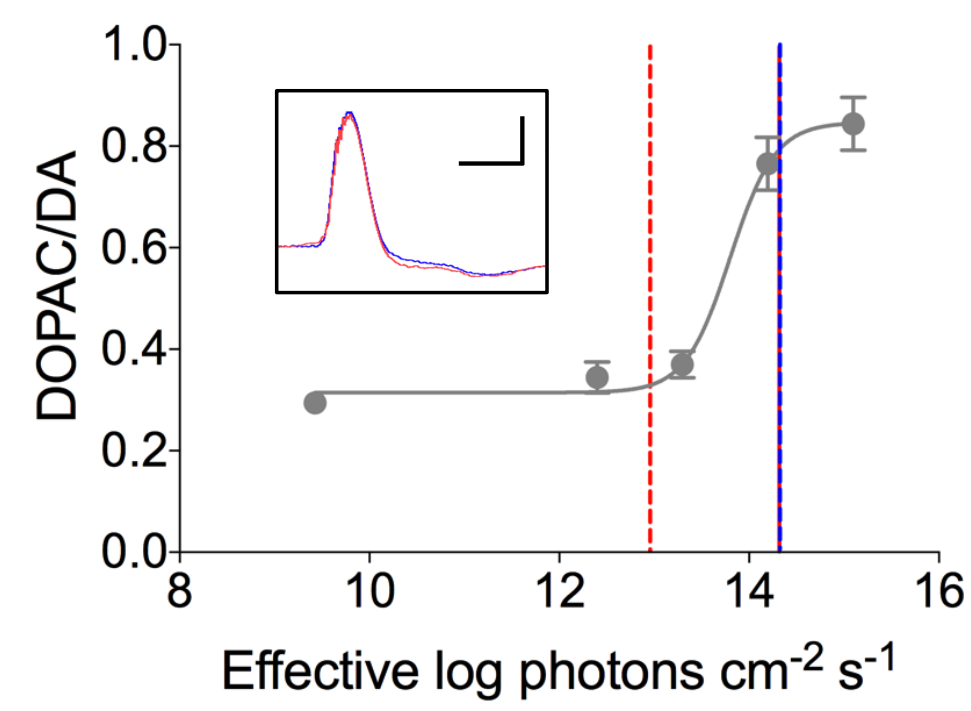

L cones

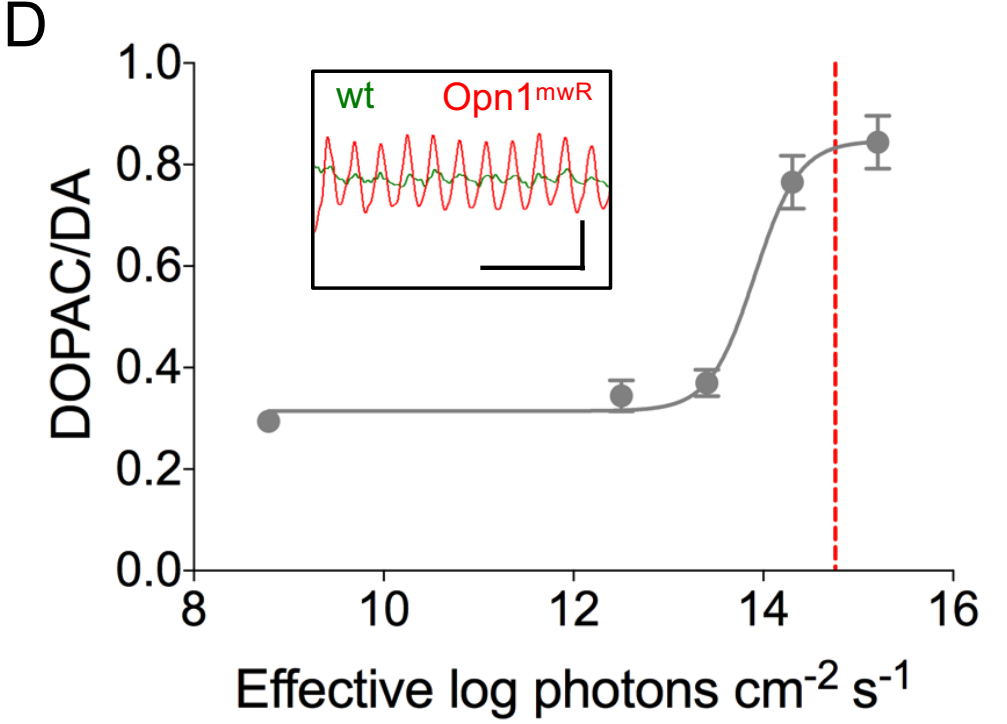

$\mathrm{E}$

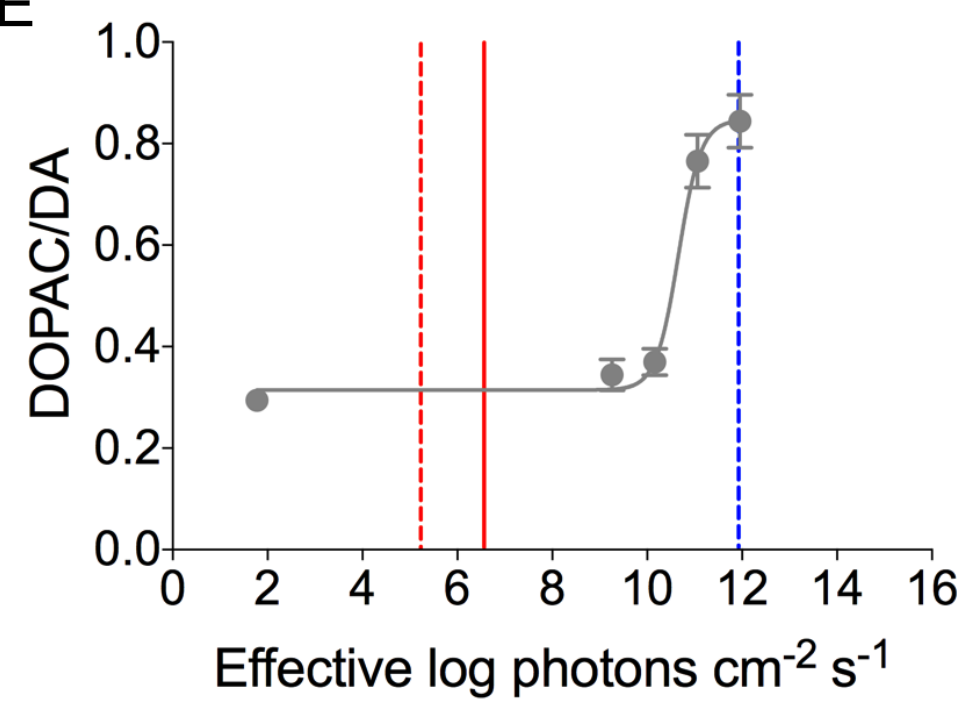

Rods

C M cones

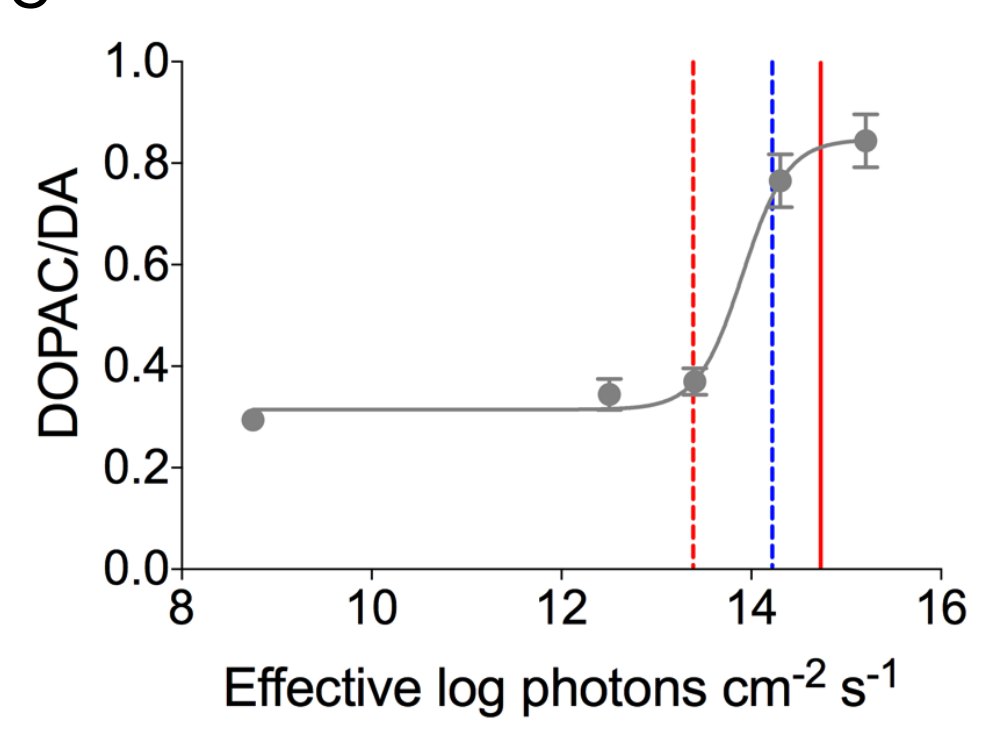

Melanopsin

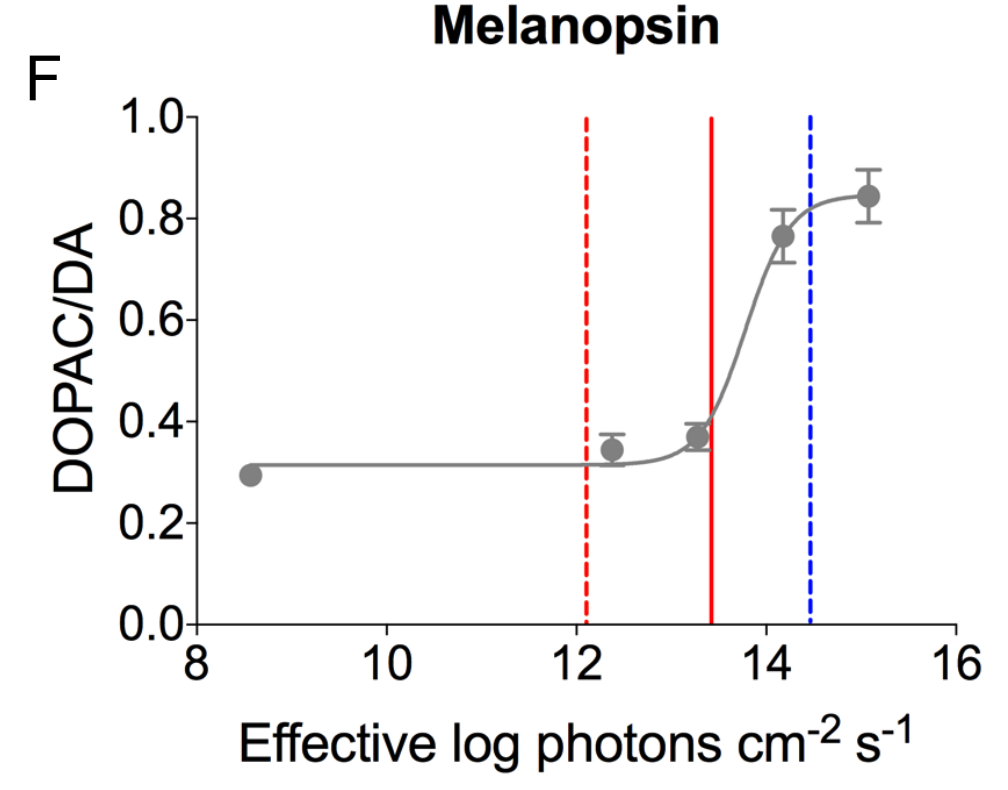

Figure S2: Red and blue stimuli. Related to Figure 2.

A) Top: dotted red line - red light pulse $(624 \mathrm{~nm} ; 15 \mathrm{~min})$ used in Figure 2G. Bottom: red solid and blue dotted lines - rod matched stimuli used in Figure $2 \mathrm{H}$.

B-F) Theoretical dopamine release curves in response to white light plotted against effective log photons $\mathrm{cm}^{-2} \mathrm{~s}^{-1}$ assuming this response is driven by B - rods $(498 \mathrm{~nm}), \mathrm{C}-\mathrm{M}$-cones $(508 \mathrm{~nm}), \mathrm{D}-\mathrm{L}$-cones $(556 \mathrm{~nm}), \mathrm{E}-\mathrm{UV}$-cones $(360 \mathrm{~nm})$ or F melanopsin (480 nm $\lambda_{\max }$ ). Red and blue pulse effective log photons $\mathrm{cm}^{-2} \mathrm{~s}^{-1}$ for each photoreceptor is plotted on each individual graph. Inserts: B, ERG response in a wild-type mouse to a dim version of the rod matched red and blue stimuli (10 rod effective log photons $\mathrm{cm}^{-2} \mathrm{~s}^{-1}$; below cone threshold; scale bar $200 \mathrm{~ms}, 50 \mu \mathrm{V}$ ). D, ERG flicker $(40 \mathrm{~Hz})$ response of wild-type (green) and Opn1 ${ }^{\mathrm{mwR}}$ (red) mice to the red light pulse (dotted red; scale bar $100 \mathrm{~ms}, 10 \mu \mathrm{V}$ ). 

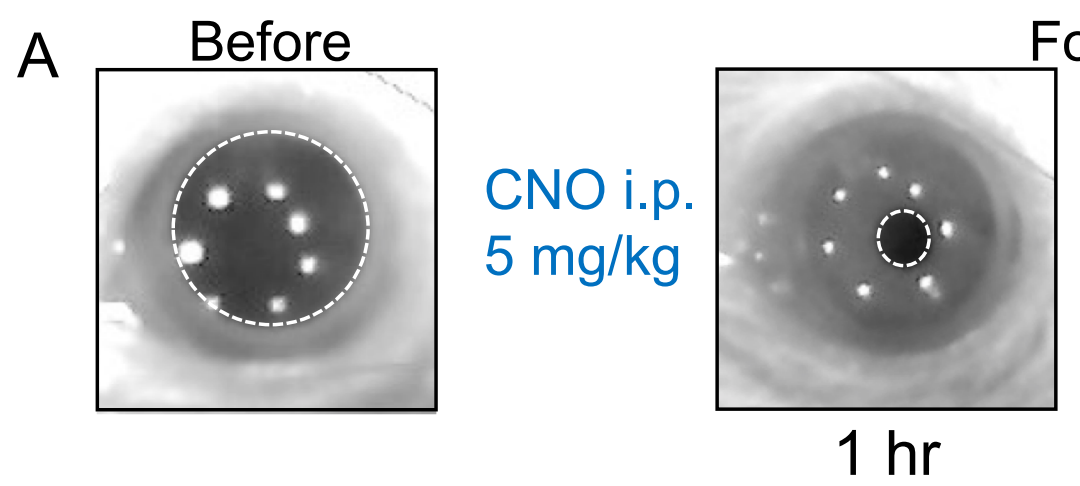

Following injection

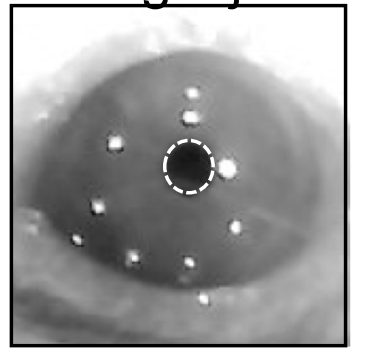

$2 \mathrm{hr}$

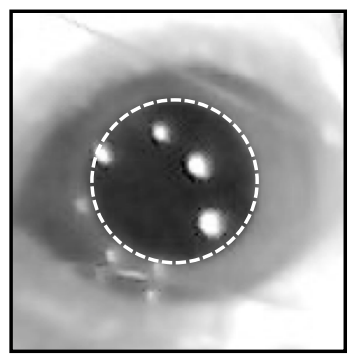

$4 \mathrm{hr}$

\section{B}

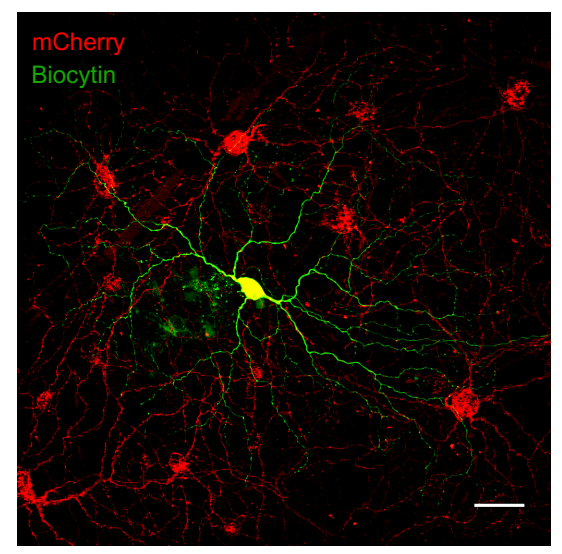

C

CNO puff $(10 \mu \mathrm{M})$

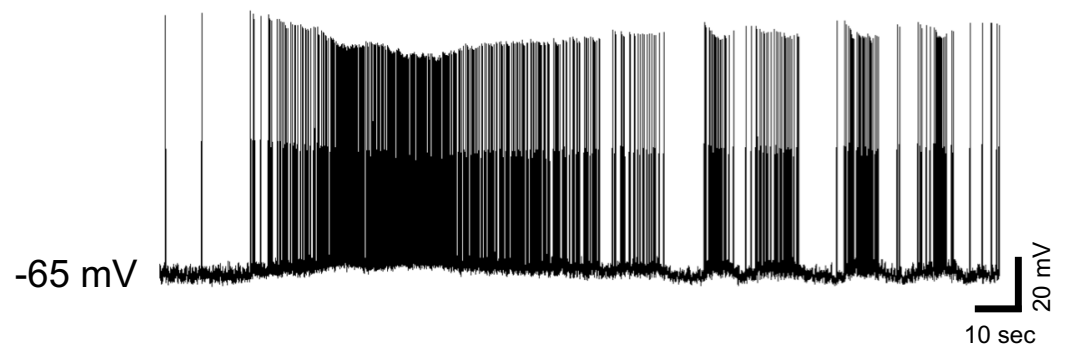

CNO bath application (10 nM)

$\mathrm{D}$

$-60 \mathrm{mV}$
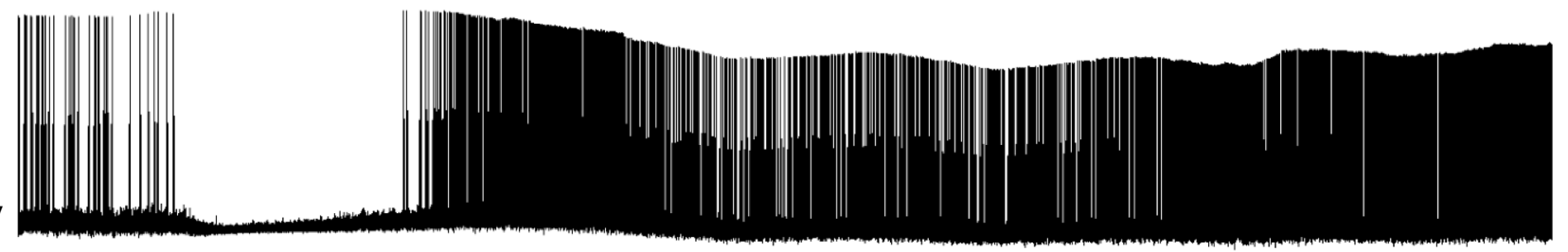

Figure S3: Activation of mRGCs in Opn $4^{\text {cre/+ }} \mathrm{hM} 3 \mathrm{Dq}$-infected animals with clozapine $\mathrm{N}$ oxide (CNO). Related to Figure 3.

A) Intraperitoneal injection (i.p.) of CNO causes prolonged pupil constriction under infrared illumination (>950 nm) that begins to subside $4 \mathrm{hrs}$ after injection.

B) hM3Dq-expressing cells can be visualized by mCherry (red) expression. Yellow/green cell shows both mCherry expression and biocytin (green) following patch-clamp recording (scale bar $30 \mu \mathrm{m}$ ).

C) Current-clamp recording from yellow/green cell in B shows that a local puff of $10 \mu \mathrm{M}$ CNO causes rapid depolarisation and spiking that decreases following the puff.

D) Slow bath application of $10 \mathrm{nM}$ CNO initially causes neuronal silencing for $~ 2.5 \mathrm{~min}$, followed by a large increase in firing rate that was maintained for the recording ( $>60 \mathrm{~min}$; trace truncated). 